\title{
Global and regional estimates of cancer mortality and incidence by site: II. results for the global burden of disease $\mathbf{2 0 0 0}$ Kenji Shibuya*1, Colin D Mathers ${ }^{1}$, Cynthia Boschi-Pinto ${ }^{2}$, Alan D Lopez ${ }^{1}$ and Christopher JL Murray ${ }^{3}$
}

Address: ${ }^{1}$ Global Program on Evidence for Health Policy, World Health Organization, Geneva, Switzerland, ${ }^{2}$ Family and Community Health/ Child and Adolescent Health and Development, World Health Organization, Geneva, Switzerland and ${ }^{3}$ Executive Director, Evidence and Information for Policy, World Health Organization, Geneva, Switzerland

Email: Kenji Shibuya* - shibuyak@who.int; Colin D Mathers - mathersc@who.int; Cynthia Boschi-Pinto - pintoc@who.int; Alan D Lopez - lopeza@who.int; Christopher JL Murray - murrayc@who.int

* Corresponding author

Published: 26 December 2002

BMC Cancer 2002, 2:37
Received: II October 2002

Accepted: 26 December 2002

This article is available from: http://www.biomedcentral.com/I47/-2407/2/37

(C) 2002 Shibuya et al; licensee BioMed Central Ltd. This is an Open Access article: verbatim copying and redistribution of this article are permitted in all media for any purpose, provided this notice is preserved along with the article's original URL.

\begin{abstract}
Background: Mortality estimates alone are not sufficient to understand the true magnitude of cancer burden. We present the detailed estimates of mortality and incidence by site as the basis for the future estimation of cancer burden for the Global Burden of Disease 2000 study.

Methods: Age- and sex- specific mortality envelope for all malignancies by region was derived from the analysis of country life-tables and cause of death. We estimated the site-specific cancer mortality distributions from vital records and cancer survival model. The regional cancer mortality by site is estimated by disaggregating the regional cancer mortality envelope based on the mortality distribution. Estimated incidence-to-mortality rate ratios were used to back calculate the final cancer incidence estimates by site.
\end{abstract}

Results: In 2000, cancer accounted for over 7 million deaths (I3\% of total mortality) and there were more than 10 million new cancer cases world wide in 2000 . More than $60 \%$ of cancer deaths and approximately half of new cases occurred in developing regions. Lung cancer was the most common cancers in the world, followed by cancers of stomach, liver, colon and rectum, and breast. There was a significant variations in the distribution of site-specific cancer mortality and incidence by region.

Conclusions: Despite a regional variation, the most common cancers are potentially preventable. Cancer burden estimation by taking into account both mortality and morbidity is an essential step to set research priorities and policy formulation. Also it can used for setting priorities when combined with data on costs of interventions against cancers.

\section{Background}

Mortality estimates alone are not sufficient to understand the true magnitude and trends of cancer problems and to evaluate the interventions against cancer, in particular preventive programmes [1]. Although cancer is still a fatal disease in many developing countries[2], the disability among cancer survivors should be taken into account since there is a growing evidence that cancer survival in some developing countries are continuously improving $[3,4]$ and, when setting priorities, cancer control interventions are compared with other health interventions which aims at reducing morbidity [5]. For this reason, estimates 
of both mortality and incidence of cancer by region are essential inputs for setting research and intervention priorities in cancer control policies.

The present study is aimed at estimating the magnitude of global and regional cancer mortality and incidence as a part of detailed analysis of all-cause levels and cause of death distributions for 191 Member States of the World Health Organization (WHO) for the Global Burden of Disease 2000 (GBD 2000) study [6]. GBD 2000 employs a time-based composite measure of disease burden in terms of disability-adjusted life years (DALYs) which consist of years lived with disability (morbidity) and years of life lost (mortality). To estimate the burden from each disease sequelae, detailed information of incidence, duration, and mortality by age, sex and region is required [5]. This study serves as a basis for the future estimation of cancer burden for the GBD 2000.
The GBD 2000 project classified WHO's 6 regions into 17 sub regions according to the levels of child and adult mortality (Table 1). On the basis of available published information on age-, sex-, and site-specific cancer incidence and survival, we developed an algorithm to estimate region-specific overall cancer mortality, and site-specific survival, death distributions, and incidence for the year 2000.

In the previous paper, we presented the cancer survival model as a key input to estimate the distribution of cancer deaths by site for the regions where mortality data are either scarce or unavailable and tested its performance and validity [7]. In this paper, we present a detailed approach to estimating mortality and incidence of cancer by site and the results of mortality and incidence estimation by site for the year 2000 .

Table I: Global Burden of Disease 2000 (GBD 2000) project: regions and sub regions

\begin{tabular}{|c|c|c|c|}
\hline WHO region & $\begin{array}{l}\text { Mortality } \\
\text { stratum }\end{array}$ & Sub region & WHO Member States \\
\hline AFRO & $\mathrm{D}$ & AfrD & $\begin{array}{c}\text { Algeria, Angola, Benin, Burkina Faso, Cameroon, Cape Verde, Chad, Comoros, Equatorial Guinea, } \\
\text { Gabon, Gambia, Ghana, Guinea, Guinea-Bissau, Liberia, Madagascar, Mali, Mauritania, Mauritius, } \\
\text { Niger, Nigeria, Sao Tome And Principe, Senegal, Seychelles, Sierra Leone, Togo, Djibouti, Somalia, } \\
\text { Sudan }\end{array}$ \\
\hline AFRO & $\mathrm{E}$ & AfrE & $\begin{array}{c}\text { Botswana, Burundi, Central African Republic, Congo, Côte d'Ivoire, Democratic Republic Of The } \\
\text { Congo, Eritrea, Ethiopia, Kenya, Lesotho, Malawi, Mozambique, Namibia, Rwanda, South Africa, } \\
\text { Swaziland, Uganda, United Republic of Tanzania, Zambia, Zimbabwe }\end{array}$ \\
\hline AMRO & A & AmrA & Canada, United States Of America \\
\hline AMRO & B & AmrB & $\begin{array}{c}\text { Antigua And Barbuda, Argentina, Bahamas, Barbados, Belize, Brazil, Chile, Colombia, Costa Rica, } \\
\text { Dominica, Dominican Republic, El Salvador, Grenada, Guyana, Honduras, Jamaica, Mexico, Panama, } \\
\text { Paraguay, Saint Kitts And Nevis, Saint Lucia, Saint Vincent And The Grenadines, Suriname, Trinidad } \\
\text { And Tobago, Uruguay, Venezuela Cuba }\end{array}$ \\
\hline AMRO & $\mathrm{D}$ & AmrD & Bolivia, Ecuador, Guatemala, Haiti, Nicaragua, Peru \\
\hline EMRO & B & EmrB & $\begin{array}{c}\text { Bahrain, Cyprus, Iran (Islamic Republic Of), Jordan, Kuwait, Lebanon, Libyan Arab Jamahiriya, Oman, } \\
\text { Qatar, Saudi Arabia, Syrian Arab Republic, Tunisia, United Arab Emirates }\end{array}$ \\
\hline EMRO & $\mathrm{D}$ & EmrD & Egypt, Iraq, Morocco, Yemen \\
\hline EURO & A & EurA & $\begin{array}{c}\text { Andorra, Austria, Belgium, Croatia, Czech Republic, Denmark, Finland, France, Germany, Greece, } \\
\text { Iceland, Ireland, Israel, Italy, Luxembourg, Malta, Monaco, Netherlands, Norway, Portugal, San } \\
\text { Marino, Slovenia, Spain, Sweden, Switzerland, United Kingdom }\end{array}$ \\
\hline EURO & $\mathrm{BI}$ & EurBI & $\begin{array}{c}\text { Albania, Bosnia And Herzegovina, Bulgaria, Georgia, Poland, Romania, Slovakia, The Former Yugo- } \\
\text { slav Republic Of Macedonia, Turkey, Yugoslavia }\end{array}$ \\
\hline EURO & B2 & EurB2 & Armenia, Azerbaijan, Kyrgyzstan, Tajikistan, Turkmenistan, Uzbekistan \\
\hline EURO & C & EurC & $\begin{array}{c}\text { Belarus, Estonia, Hungary, Kazakhstan, Latvia, Lithuania, Republic of Moldova, Russian Federation, } \\
\text { Ukraine }\end{array}$ \\
\hline SEARO & B & SearB & Indonesia, Sri Lanka, Thailand Malaysia, Philippines Brunei Darussalam, Singapore \\
\hline SEARO & $\mathrm{D}$ & SearD & Bangladesh, Bhutan, India, Maldives, Nepal Afghanistan, Pakistan \\
\hline WPRO & A & WprA & Australia, Japan, New Zealand \\
\hline WPRO & BI & WprBI & China, Mongolia, Republic Of Korea DPR Korea \\
\hline WPRO & B2 & WprB2 & Cambodia, Lao People's Democratic Republic, Viet Nam Myanmar \\
\hline WPRO & B3 & WprB3 & $\begin{array}{c}\text { Cook Islands, Fiji, Kiribati, Marshall Island s, Micronesia (Federated States Of), Nauru, Niue, Palau, } \\
\text { Papua New Guinea, Samoa, Solomon Islands, Tonga, Tuvalu, Vanuatu }\end{array}$ \\
\hline
\end{tabular}




\section{Material and Methods Overview of analysis}

The Global Burden of Disease 2000 (GBD 2000) study deals with the problem of systemic bias in cause-specific mortality by estimating total mortality for each Member State, starting from an analysis of the overall mortality envelope for each region, in order to ensure that the causespecific estimates add to the total all cause mortality by age and sex and that there is not systematic over- or underestimation or double counting of deaths. This regional cause-specific mortality envelope serves as an upper bond of mortality from a certain cause and partly ensures the internal consistency among incidence, prevalence and mortality rates [6]. The approaches to estimating mortality and incidence of cancer by site also follows this process (Figure 1).

Firstly, we obtained the age- and sex- specific mortality envelope for all malignancies by region derived from the analysis of country life-tables and cause of death models in each region. Secondly, we estimated the site-specific cancer mortality distributions from vital records or cancer survival models depending on the availability and quality of detailed cause of death data. Cancer sites for which survival was calculated were: mouth and pharynx (ICD-10 C00-C14), oesophagus (C15), stomach (C16), colon and rectum (C18-C21), liver (C22), pancreas (C25), trachea, bronchus and lung (C33-C34), melanoma (C43), female breast (C50), cervix uterine (C53), corpus uteri (C54-55), ovary (C56), prostate (C61), bladder (C67), lymphomas and multiple myeloma (C81-C90, C96), leukaemia (C91C95), and other malignant neoplasms (balance of ICD-10 C00-C97). The GBD 2000 assigns Kaposi's sarcoma and non-Hodgkin lymphomas (NHL) attributable to HIV/ AIDS among AIDS sequela and their burden is included separately with HIV/AIDS. Thirdly, the final regional cancer mortality by site is estimated by disaggregating the regional cancer envelope based on the mortality distribution by site. Finally, we applied the incidence-tomortality rate ratios to back calculate the final cancer incidence estimates by site.

\section{GBD 2000 regional mortality estimates by age, sex, and cause}

Complete or incomplete vital registration data together with sample registration systems now cover $74 \%$ of global mortality in 128 countries. Survey data and indirect demographic techniques provide information on levels of child and adult mortality for the remaining $26 \%$ of estimated global mortality. The available sources of mortality data for the WHO sub regions of the GBD 2000 are summarised in Table 1.

Causes of death for the WHO sub regions and the world have been estimated based on data from national vital registration systems that capture about 17 million deaths annually. In addition, information from sample registration systems, population laboratories and epidemiological analyses of specific conditions have been used to improve estimates of the cause of death patterns $[6,8,9]$. As a general rule, vital registration data, suitably corrected for ill-defined coding and probable systematic biases in certifying deaths to non-specific vascular, cancer and injury codes were used to estimate the cause of death pattern. The GBD 2000 approach adjusts the cause-specific mortality to include a proportion of deaths coded to ill-defined causes in vital registration data and redistributes these deaths pro-rata among broader communicable and non-communicable causes.

Cause of death data have been carefully analysed to take into account incomplete coverage of vital registration in countries and the likely differences in cause of death patterns that would be expected in the uncovered and often poorer sub-populations [10]. For countries lacking sufficient vital registration data, cause of death models were used to firstly estimate the maximum likelihood distribution of deaths across the broad categories of communicable, non-communicable and injuries, based on estimated total mortality rates and income [8]. A regional model pattern of specific causes of death was then constructed based on local vital registration and verbal autopsy data, and this proportionate distribution was then applied within each broad cause group. Finally, the resulting estimates were then adjusted based on other epidemiological evidence from specific disease studies. Methods used to estimate global all-cause and cause-specific mortality from these data are described elsewhere [6].

In the GBD 1990 [5], deaths coded to ICD-9 195-199 (i.e., malignant neoplasm of other and unspecified sites including those whose point of origin cannot be determined, secondary and unspecified neoplasm) were redistributed pro-rata across all malignant neoplasm categories within each age-sex group, so that the category 'Other malignant neoplasms' includes only malignant neoplasms of other specified sites. Reviews of the cancer registry and methods of diagnosis suggest that four sites (month and pharynx, liver, breast and cervix uteri) where there did not appear to be any significant mis-coding of cancer deaths, since these can be diagnosed clinically without any further diagnostic tests even in developing regions [11]. Accordingly, the cancer garbage code redistribution algorithm was revised for the GBD 2000 to redistribute cancer deaths in ICD C76-C80 pro-rata across the sites other than the four sites above.

\section{Estimation of cancer mortality and incidence}

Given the regional cancer mortality envelope by age and sex, the site-specific distributions of cancer mortality are 


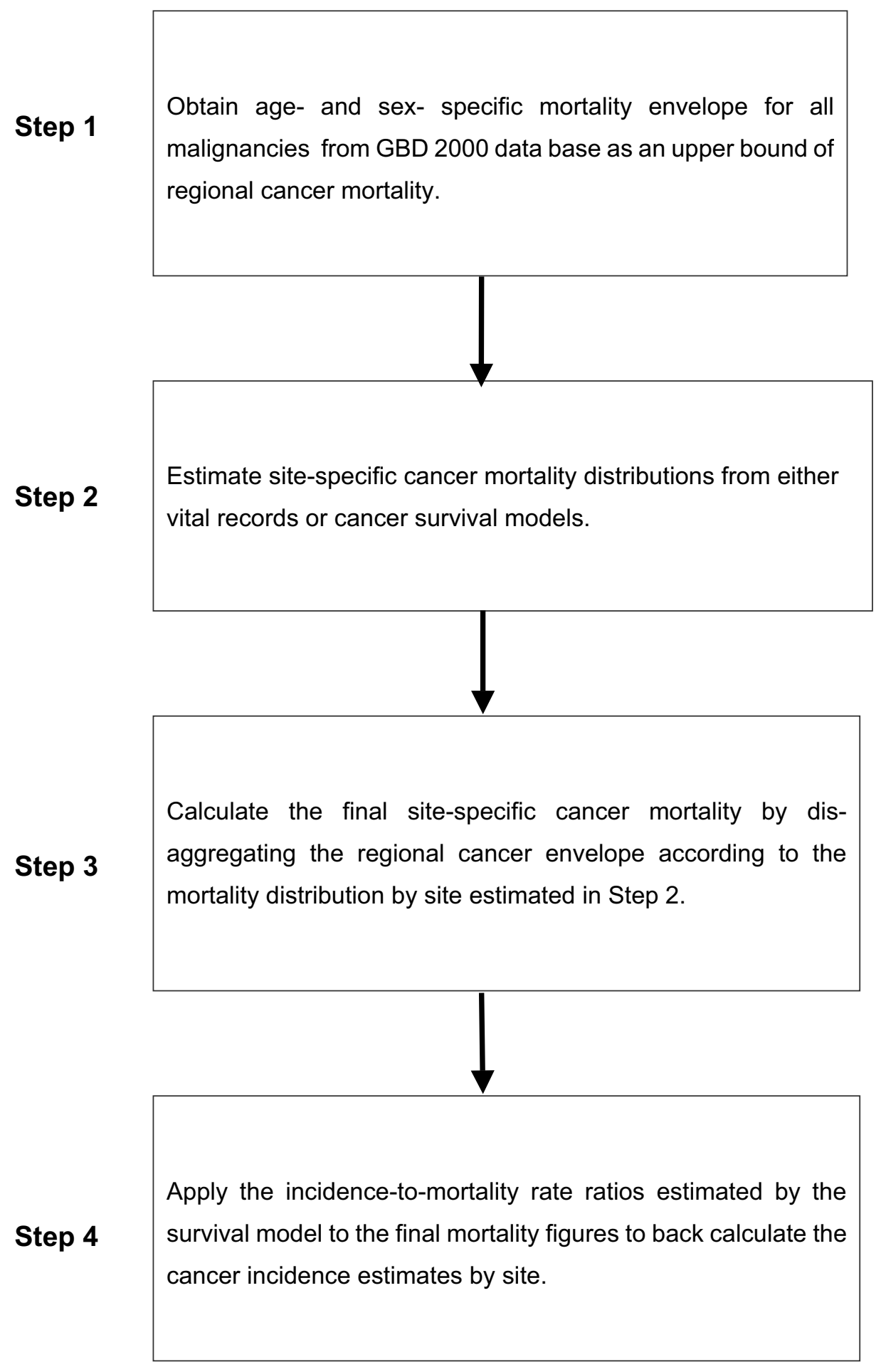

Figure I

Approaches to estimating global and regional cancer mortality and incidence by site for the Global Burden of Disease 2000 (GBD 2000) 
necessary to disagreggate the estimated total cancer deaths by age and sex for each region. The approaches to estimating mortality distributions were different depending on the availability and quality of data on detailed causes of death.

Direct estimates of the site-specific distributions of cancer mortality were possible for the regions where established vital registration records with high coverage and ICD-coding are available, including countries in the A sub regions (AmrA, EurA and WprA) and countries in AmrB, EurB1, EurB2 and EurC [6]. For the other regions of the world (AfrD, AfrE, AmrD, EmrB, EmrD, SearB, SearD, WprB1, WprB2 and WprB3 sub regions), a site-specific model for relative interval survival adjusted for each region was developed and applied to the regional incidence estimated by International Agency for Research on Cancer (IARC) to calculate the mortality distribution by site for the year $2000[7,12]$. The region-specific incidence estimates of the Globocan 2000 was adjusted to ensure the consistency with the GBD 2000 definitions [7]. Our survival model and adjusted incidence estimates of the Globocan 2000 were primarily used to estimate the distribution, but not the magnitude, of cancer by site, sex, and age group.

The final site-specific cancer mortality was then estimated by multiplying age- and sex- specific regional cancer mortality envelope by estimated cancer mortality distribution by site. The cancer survival model is flexible enough to yield the survival estimates of various age, years and period as well as mean duration of time of cancer by site [7]. We estimated incidence-to-mortality rate ratios at each age group form the survival model and, by using the final cancer mortality, applied the ratios to back calculate the final incidence by age and sex for each cancer site in all regions of the world for the year 2000 .

\section{Results}

\section{Cancer mortality by region}

The GBD 2000 classifies the cause of deaths into three broad categories: communicable disease, non-communicable disease, and injuries. In 2000, approximately 56 million deaths occurred world wide and non-communicable disease accounted for $58 \%$ of total number of deaths, followed by communicable disease (33\%) and injuries (9\%) (Figure 2). Cancer was estimated to account for over 7 million deaths (13\% of total mortality and $22 \%$ of non-communicable disease mortality) world wide in 2000, only preceded by cardiovascular diseases (29\% of total) and infectious and parasitic diseases (19\%). Depending on the extent of health transition, cancer mortality as a proportion of total mortality differed substantially by region, from less than 5\% in AfrE to approximately $30 \%$ in WprA sub region.
Tables 2,3,4,5 show the regional estimates of total number of deaths and age-specific mortality rates of all cancers for the GBD 2000. More than $60 \%$ of cancer deaths occurred in developing regions, particularly in SearD (mainly India) and WprB1(mainly China) sub regions due to their large population size. On average global cancer mortality rates among males and females were 128.2 and 104.6 per 100,000, respectively. Not surprisingly crude mortality rates from all cancers vary significantly and are much higher in developed regions (AmrA, EurA, and WprA). However, since age-specific mortality rate is generally higher in developing regions, regional mortality rates, age-standardised to the world population, showed less variations compared to crude mortality rates and higher mortality rates were observed in regions such as AfrD, AfrE, AmrB, EurC, and WprB (Figure 3).

Cancer mortality envelope was disaggregated by applying the distribution of cancer mortality by site estimated from both the detailed analysis of vital records and the use of survival model and incidence data from the International Agency for Research on Cancer (IARC). Tables 6 and 7 show the estimated global and regional numbers of deaths and age-standardised mortality rates by sex for 17 cancer sites. Generally higher mortality rates were observed in developing regions except cancers of lung and pancreas which are more prevalent in developed regions and highly fatal.

Tables 8 and 9 represent the ranking of the number of deaths by cancer site in the world and three selected sub regions: a high child and adult mortality/low income region (AfrE), a very low child and adult mortality/high income region (EurA), and a low child and adult mortality/ middle income region (SearB). Lung cancer was the leading cause of cancer deaths in the world, accounting for $17 \%$ of total cancer mortality, followed by cancers of stomach $(12 \%$ of total), liver $(9 \%)$, colon and rectum $(9 \%)$, and breast (7\%). In males, lung, stomach, and liver cancers were the three most common cause of cancer deaths. The leading cause of cancer deaths among females was breast but lung cancer was already the second largest cause of cancer deaths.

There is a significant variation in the distribution of sitespecific cancer mortality by region. In AfrE, the leading causes of cancer mortality in males and females were liver and cervix uteri cancers, respectively, both of which are primarily due to viral infections. Liver cancer accounted for approximately one quarter of all male cancer deaths and cervical cancer accounted for more than one-third of female cancer deaths. In EurA, the pattern of cancer mortality distribution is a typical one observed in industrialised countries: lung, colorectal, and prostate cancers accounted for a large proportion of male cancer mortality 


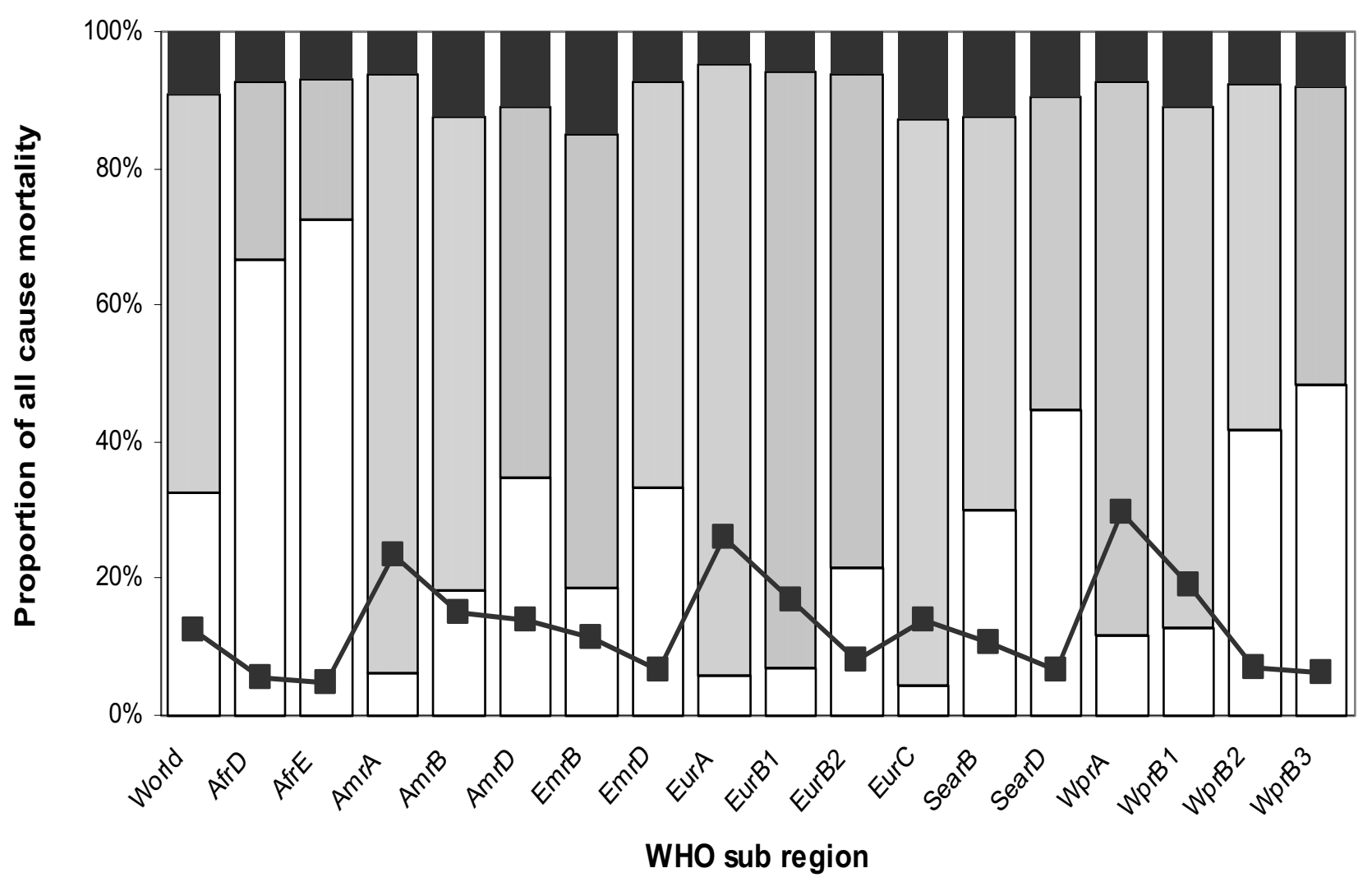

Communicable disease $\square$ Non-communicable disease Injuries $\rightarrow$-Cancer

Figure 2

Regional distribution of mortality by cause, 2000

while breast, colorectal, and lung cancers were major causes among females. Site-specific cancer mortality distribution in SearB lay somewhere between the two sub regions, although lung and breast cancers were already leading causes of cancer deaths in males and females, respectively.

\section{Cancer incidence by site}

Regional incidence was back calculated from multiplying mortality by site-specific incidence-to-mortality rate ratios by age, sex, and region. As shown in Table 10, (age-standardised) incidence-to-mortality rate ratio was generally higher in cancers with relatively better prognosis including lymphoma, leukaemia, and cancers of colon and rectum, breast, uterus, and ovary. As a result, the new cases of such cancers as a proportion of total new cases were higher than the proportion of mortality from same cancers. Although this pattern was consistent across the regions, the ratios varied substantially by age, sex, and region (data not shown).

Tables 11,12,13 show the regional estimates of total new cases and age-specific incidence rates of all cancers in 2000. More than 10 million new cancers cases occurred world wide. Due to the differences in survival by region, estimated incidence-to-mortality rate ratios were generally much higher in developed regions and the proportion of cancer incidence in developing regions was approximately $50 \%$ of total cancer incidence in the world. Despite their much smaller population size, the number of new cases in AmrA and EurA are comparable to those in SearD and WprB1. On average global cancer incidence rates among males and females were 169.7 and 171.1 per 100,000 , respectively (Figure 4). Regional age-standardised incidence rates also suggest that female incidence was slightly higher than male incidence in developing regions, particularly in 


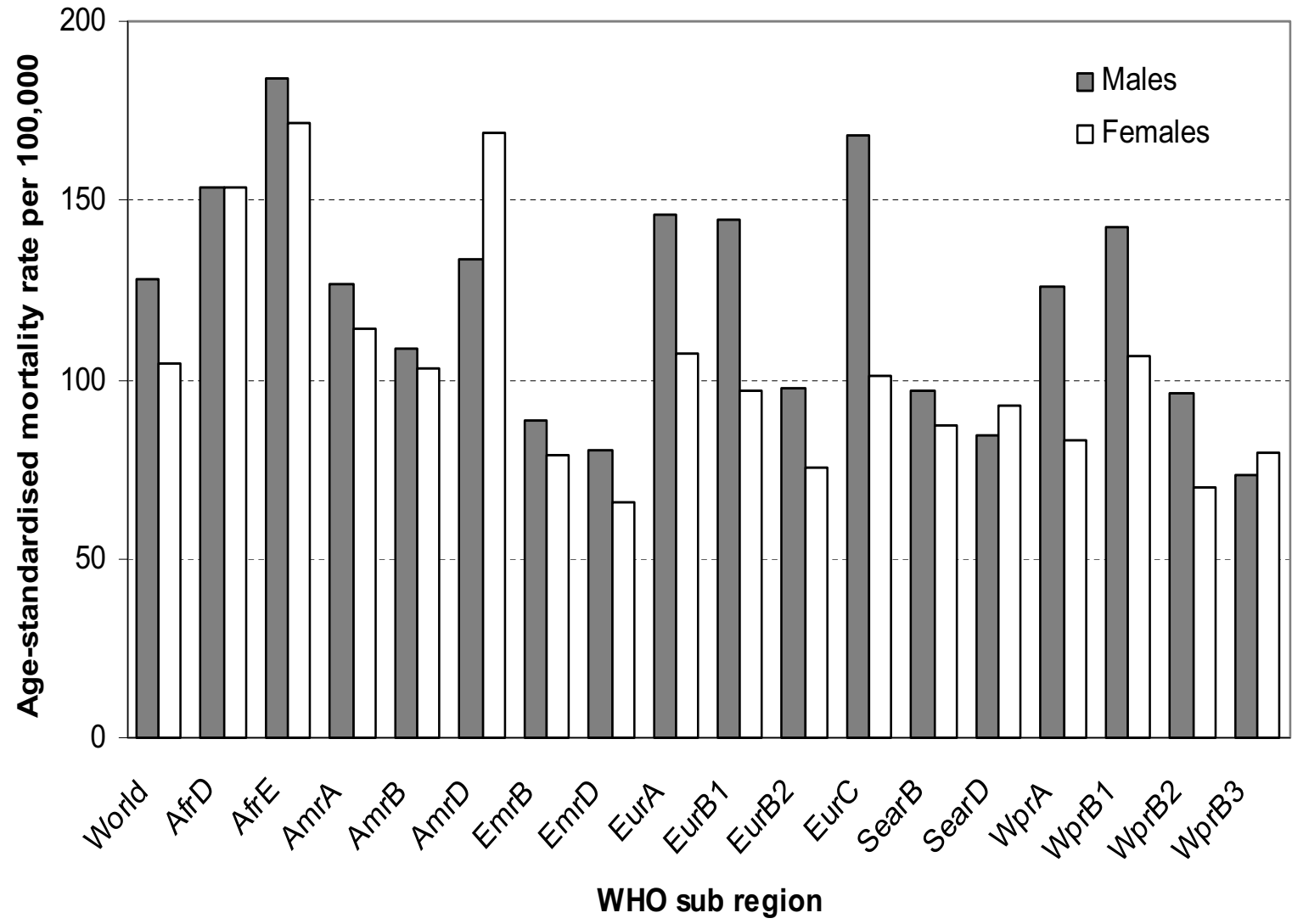

Figure 3

Age-standardised mortality rate from all cancers by region, 2000

AmrD where the number of deaths female cancers was high. On the other hand, male and female incidence rates were almost the same in all A sub regions (AmrA, EurA, and WprA).

Estimated global and regional numbers of new cases and age-standardised incidence rates for 17 cancer sites by sex are shown in Tables 14 and 15. As in mortality distribution, there is a significant variations in the distribution of site-specific cancer incidence by region (Tables 16 and 17). The distribution of cancer incidence was almost similar to that of mortality: lung cancer was the most common cancer in the world in 2000, accounting for $13 \%$ of total cancer mortality, followed by cancers of stomach (10\% of total), liver (10\%), colon and rectum (10\%), and breast $(6 \%)$. The variations in the distribution of site-specific new cases of cancer by region were also similar to those observed in mortality distribution. In all regions, compared to proportion of total mortality, the proportion of lung cancer decreased while that of colon and rectum, breast, uterus and prostate increased, suggesting the difference in survival by cancer site as suggested above.

\section{Discussion}

Various attempts have been made to quantify the global burden of cancer and to estimate site-specific cancer mortality and morbidity [1,12-15]. More recently, the efforts made by the International Agency for Research on Cancer (IARC) have led to the Globocan 2000 estimates, which has also used information on incidence and survival to estimate cancer death for the year 2000 from various sources including cancer registries [12,13]. The analyses reported here have built extensively on the IARC work to synthesis and estimate cancer mortality and incidence distributions by site for all regions of the world. Compared to the estimates of the Globocan 2000, the GBD 2000 estimates 


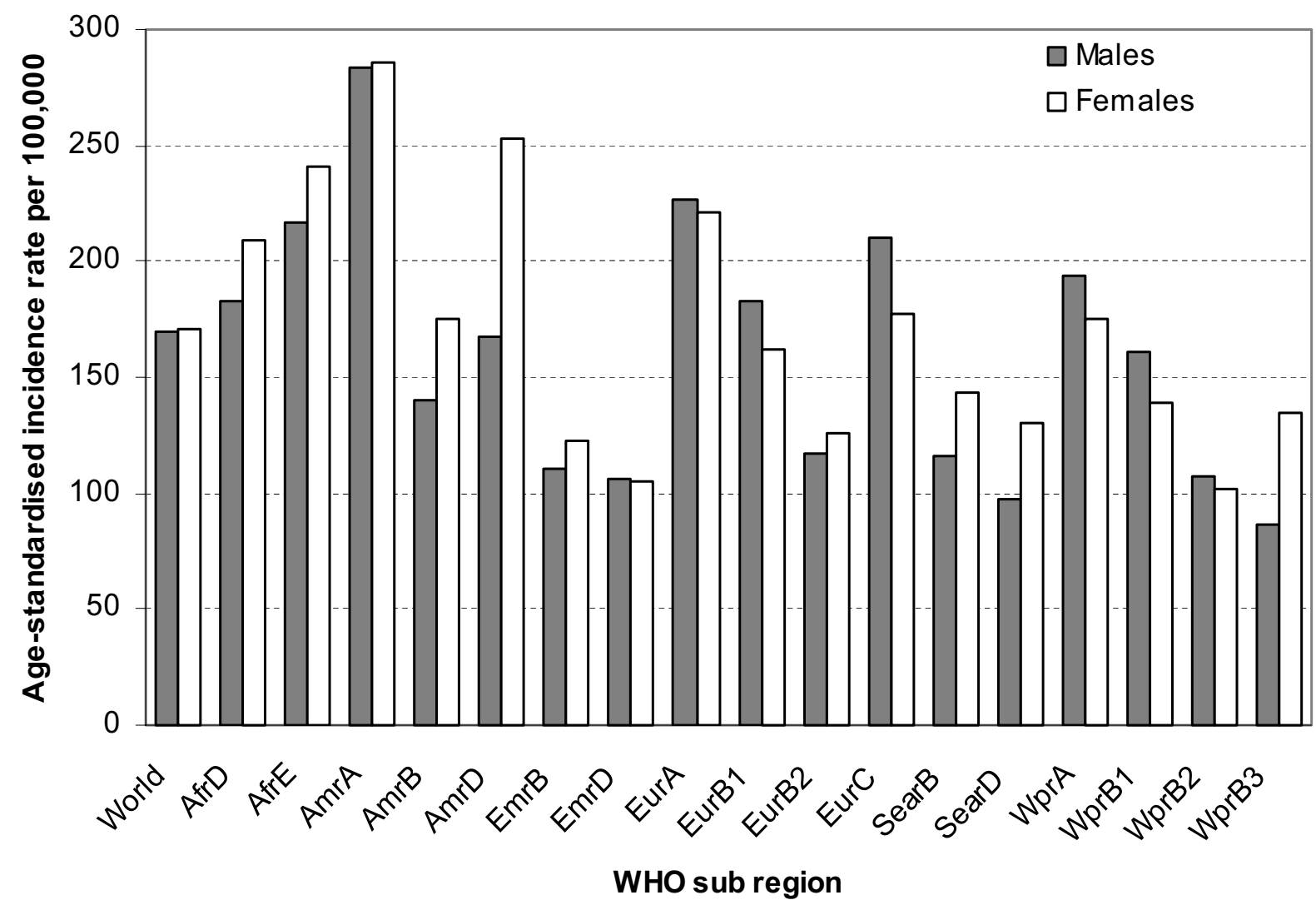

Figure 4

Age-standardised incidence rate from all cancers by region, 2000

for global cancer mortality and incidence are $11 \%$ and 3\% higher, respectively. Although the overall difference in terms of proportion is small, the absolute difference between the two estimates remains relatively large. This difference is predominantly due to a substantially large difference in the AFRO, EMRO, and SEARO regions.

Some researchers suggest that model-based estimates of cancer mortality in GBD 1990 bear little relation to the actual profile of cancer recorded the regional registries $[1,16]$. The proposed approach here is substantially different from the previous estimates and, although broader cause of death to estimate cancer envelope is still based on cause of death models in some countries, majority of the data sources of cause of death is now vital registration and/or sampling data. The total number of mortality from cancer is not extrapolated by model alone and the survival model was used to estimate the distribution of death by site, not the actual magnitude of cancer mortality in regions where no or little data on detailed cause of death is available. The model estimates were consistent with mortality distribution of vital records and the Globocan 2000 [7]. Therefore, the major source of discrepancy is not the estimated cancer mortality distribution but the overall mortality envelope applied to each region.

The Globocan 2000 estimates are based on either cancer incidence data from cancer registries in the region (with a survival model used to estimate deaths) or on mortality data collected by regional cancer registries or other sources. Both these sources of data are likely to be incomplete and to result in underestimation of cancer deaths. On the other hand, the GBD 2000 starts with data on the level of all-cause mortality, and uses all available data on cause of death and cause of death models where such data is not available, to estimate the distribution of major 
Table 2: Mortality data sources (number of Member States with recent deaths coverage) by WHO sub region for the GBD2000

\begin{tabular}{|c|c|c|c|c|c|c|}
\hline Subregion & $\begin{array}{l}\text { Category I: Com- } \\
\text { plete vital statistics } \\
(95 \%+\text { coverage })\end{array}$ & $\begin{array}{l}\text { Category II: } \\
\text { Incomplete vital } \\
\text { statistics }\end{array}$ & $\begin{array}{l}\text { Category III: Sam- } \\
\text { ple registration and } \\
\text { surveillance systems }\end{array}$ & $\begin{array}{l}\text { Category IV: } \\
\text { Child mortality esti- } \\
\text { mated from sur- } \\
\text { veys and censuses }\end{array}$ & $\begin{array}{l}\text { Category V: No } \\
\text { recent data on child } \\
\text { or adult mortality }\end{array}$ & $\begin{array}{l}\text { Number of } \\
\text { countries }\end{array}$ \\
\hline AfrD & 2 & 2 & 0 & 19 & 3 & 26 \\
\hline AfrE & 0 & 2 & 1 & 14 & 3 & 20 \\
\hline AmrA & 3 & 0 & 0 & 0 & 0 & 3 \\
\hline $\mathrm{AmrB}$ & 17 & 9 & 0 & 0 & 0 & 26 \\
\hline AmrD & 0 & 4 & 0 & 2 & 0 & 6 \\
\hline EmrB & 4 & 3 & 0 & 6 & 0 & 13 \\
\hline EmrD & 0 & 2 & 0 & 5 & 2 & 9 \\
\hline EurA & 26 & 0 & 0 & 0 & 0 & 26 \\
\hline EurB & 7 & 9 & 0 & 0 & 0 & 16 \\
\hline EurC & 8 & 1 & 0 & 0 & 0 & 9 \\
\hline SearB & I & I & 0 & 1 & 0 & 3 \\
\hline SearD & 0 & 1 & 2 & 3 & I & 7 \\
\hline WprA & 4 & 1 & 0 & 0 & 0 & 5 \\
\hline WprB & 3 & 12 & I & 6 & 0 & 22 \\
\hline World & 75 & 47 & 4 & 56 & 9 & 191 \\
\hline
\end{tabular}

Table 3: Estimated global and regional number of total cancer deaths (thousands) and mortality rate (per 100,000) by age, both sexes, 2000

\begin{tabular}{|c|c|c|c|c|c|c|c|c|c|}
\hline \multirow[t]{2}{*}{ Region } & \multicolumn{9}{|c|}{ Age-group } \\
\hline & $0-4$ & $5-14$ & $15-29$ & $30-44$ & $45-59$ & $60-69$ & $70-79$ & $80+$ & Total \\
\hline \multicolumn{10}{|c|}{ Number of deaths $(000$ s) } \\
\hline World & 46.0 & 49.4 & $|4| .3$ & 462.1 & $1,503.0$ & I,852.4 & $1,901.0$ & $\mathrm{I}, 078.7$ & $7,033.9$ \\
\hline AfrD & 5.0 & 2.5 & 10.7 & 26.3 & 59.2 & 70.0 & 66.5 & 31.5 & 271.6 \\
\hline AfrE & 4.9 & 2.6 & 15.2 & 33.1 & 65.0 & 76.4 & 72.1 & 31.5 & 300.8 \\
\hline AmrA & 0.6 & 1.2 & 3.9 & 23.1 & 102.4 & 132.3 & 194.5 & 168.1 & 626.2 \\
\hline$A m r B$ & 3.2 & 4.4 & 10.7 & 31.7 & 86.4 & 94.3 & 103.5 & 66.6 & 400.8 \\
\hline$A m r D$ & 1.0 & 1.4 & 2.7 & 6.4 & 14.8 & 16.0 & 18.9 & 11.5 & 72.9 \\
\hline EmrB & 1.2 & 2.0 & 3.8 & 8.0 & 19.2 & 18.2 & 17.8 & 7.7 & 77.8 \\
\hline EmrD & 1.9 & 2.5 & 4.3 & 10.6 & 17.8 & 15.1 & 13.2 & 4.3 & 69.7 \\
\hline EurA & 0.7 & 1.5 & 5.3 & 31.3 & I54.I & 238.2 & 350.9 & 279.8 & $1,061.7$ \\
\hline EurBI & 0.7 & I.I & 3.9 & 16.6 & 59.3 & 76.6 & 69.8 & 26.5 & 254.5 \\
\hline EurB2 & 0.2 & 0.6 & 1.4 & 4.3 & 7.9 & 10.9 & 7.5 & 1.9 & 34.8 \\
\hline EurC & 0.8 & 1.7 & 5.8 & 28.4 & 114.2 & 162.1 & 146.1 & 44.5 & 503.7 \\
\hline SearB & 2.9 & 4.0 & 7.9 & 30.9 & 90.0 & 79.8 & 59.1 & 25.9 & 300.6 \\
\hline SearD & 12.9 & 11.9 & 32.7 & 65.1 & 189.0 & 262.1 & 192.2 & 109.2 & 875.1 \\
\hline WprA & 0.2 & 0.4 & 1.6 & 7.7 & 49.6 & 77.6 & 104.2 & 90.4 & 331.6 \\
\hline WprBI & 8.9 & 10.6 & 28.5 & 128.2 & 451.6 & 503.1 & 467.5 & 165.6 & I,764.0 \\
\hline WprB2 & 0.9 & 1.0 & 3.0 & 9.5 & 21.4 & 18.9 & 16.7 & 13.3 & 84.8 \\
\hline WprB3 & 0.0 & 0.1 & 0.2 & 0.6 & 1.2 & 0.7 & 0.4 & 0.2 & 3.4 \\
\hline \multicolumn{10}{|c|}{ Age-specific mortality rate per 100,000} \\
\hline World & 7.6 & 4.1 & 9.0 & 36.3 & 186.8 & 548.2 & 965.1 & I,702.I & 116.5 \\
\hline AfrD & 9.1 & 2.8 & 11.6 & 49.5 & 210.0 & 667.6 & $1,386.4$ & $2,799.0$ & 81.4 \\
\hline AfrE & 8.5 & 2.7 & 16.3 & 63.6 & 243.8 & 798.9 & $1,612.8$ & $2,932.2$ & 89.1 \\
\hline AmrA & 2.9 & 2.6 & 6.3 & 31.5 & 178.2 & 581.8 & I,074. I & $1,901.0$ & 203.1 \\
\hline$A m r B$ & 7.0 & 4.9 & 8.6 & 34.1 & 159.9 & 463.9 & 895.3 & I,773.8 & 90.7 \\
\hline AmrD & 10.7 & 8.2 & 13.3 & 51.0 & 211.0 & 593.4 & $\mathrm{I}, 354.4$ & $2,827.8$ & 102.3 \\
\hline EmrB & 7.3 & 5.7 & 9.1 & 31.5 & $\mid 44.0$ & 374.6 & 688.6 & $1,182.8$ & 55.8 \\
\hline EmrD & 10.2 & 7.5 & 11.0 & 41.5 & 129.5 & 305.6 & 540.6 & 716.3 & 50.5 \\
\hline EurA & 3.2 & 3.1 & 6.6 & 33.1 & 196.8 & 570.4 & $1,109.9$ & $2,089.7$ & 259.6 \\
\hline
\end{tabular}


Table 3: Estimated global and regional number of total cancer deaths (thousands) and mortality rate (per 100,000$)$ by age, both sexes, 2000 (Continued)

\begin{tabular}{lllllllccc}
\hline EurBI & 5.7 & 4.2 & 9.0 & 47.1 & 226.3 & 602.5 & 874.0 & $1,325.3$ & 153.5 \\
EurB2 & 3.7 & 4.8 & 10.0 & 41.5 & 172.8 & 450.4 & 584.6 & 550.4 & 68.4 \\
EurC & 6.7 & 5.1 & 10.4 & 51.3 & 266.1 & 656.0 & 911.4 & $1,012.8$ & 205.4 \\
SearB & 7.1 & 5.0 & 7.0 & 36.7 & 192.9 & 442.1 & 679.3 & $1,109.6$ & 76.2 \\
SearD & 8.0 & 3.9 & 8.8 & 24.9 & 124.6 & 448.9 & 681.4 & $1,499.2$ & 64.9 \\
WprA & 2.4 & 2.3 & 5.1 & 25.9 & 153.3 & 471.8 & 927.3 & $1,907.7$ & 222.6 \\
WprBI & 8.6 & 4.5 & 8.4 & 38.1 & 218.2 & 617.6 & $1,080.7$ & $1,435.2$ & 130.0 \\
WprB2 & 5.9 & 3.3 & 7.2 & 31.7 & 152.4 & 312.2 & 525.6 & $1,540.4$ & 59.7 \\
WprB3 & 4.3 & 4.3 & 8.2 & 47.8 & 173.4 & 280.3 & 378.4 & $1,162.9$ & 49.1 \\
\hline
\end{tabular}

Table 4: Estimated global and regional number of total cancer deaths (thousands) and mortality rate (per 100,000) by age, males, 2000

\begin{tabular}{|c|c|c|c|c|c|c|c|c|c|}
\hline \multirow[t]{2}{*}{ Region } & \multicolumn{9}{|c|}{ Age-group } \\
\hline & $0-4$ & $5-14$ & $15-29$ & $30-44$ & $45-59$ & $60-69$ & 70-79 & $80+$ & Total \\
\hline \multicolumn{10}{|c|}{ Number of deaths (000s) } \\
\hline World & 22.6 & 27.4 & 79.9 & 228.3 & 844.8 & $1,121.7$ & $1,074.6$ & 502.6 & $3,901.9$ \\
\hline AfrD & 2.1 & 1.3 & 6.2 & 14.3 & 29.2 & 34.2 & 34.8 & 17.1 & 139.3 \\
\hline AfrE & 2.6 & 1.5 & 9.6 & 20.3 & 33.3 & 36.2 & 36.1 & 17.0 & 156.8 \\
\hline AmrA & 0.3 & 0.6 & 2.3 & 10.5 & 54.0 & 74.5 & 105.8 & 77.1 & 325.1 \\
\hline AmrB & 1.7 & 2.5 & 6.0 & 12.4 & 41.4 & 51.7 & 57.6 & 33.9 & 207.1 \\
\hline AmrD & 0.4 & 0.8 & 1.4 & 2.2 & 5.4 & 7.5 & 9.6 & 5.9 & 33.3 \\
\hline EmrB & 0.7 & 1.2 & 1.9 & 3.0 & 10.0 & 11.6 & 11.6 & 4.8 & 44.9 \\
\hline EmrD & 1.2 & 1.5 & 2.4 & 5.1 & 9.0 & 8.8 & 7.6 & 2.7 & 38.2 \\
\hline EurA & 0.4 & 0.9 & 3.2 & 15.2 & 90.1 & 151.1 & 205.8 & 127.8 & 594.3 \\
\hline EurBI & 0.4 & 0.7 & 2.1 & 8.7 & 37.2 & 49.5 & 39.9 & 12.1 & 150.5 \\
\hline EurB2 & 0.1 & 0.3 & 0.8 & 2.0 & 4.4 & 6.6 & 3.9 & 0.7 & 18.9 \\
\hline EurC & 0.4 & 1.0 & 3.3 & 13.8 & 70.1 & 101.2 & 73.5 & 15.9 & 279.2 \\
\hline SearB & 1.5 & 2.0 & 4.2 & 11.8 & 45.4 & 48.4 & 35.7 & 12.8 & 161.8 \\
\hline SearD & 6.0 & 6.0 & 15.5 & 31.0 & 88.4 & 148.3 & 106.4 & 48.7 & 450.1 \\
\hline WprA & 0.1 & 0.2 & 0.8 & 3.4 & 29.2 & 52.8 & 67.0 & 44.9 & 198.5 \\
\hline WprBI & 4.1 & 6.2 & 18.7 & 69.7 & 285.7 & 327.5 & 268.6 & 73.8 & $1,054.3$ \\
\hline WprB2 & 0.5 & 0.6 & 1.6 & 4.6 & 11.4 & 11.7 & 10.3 & 7.2 & 47.9 \\
\hline WprB3 & 0.0 & 0.0 & 0.1 & 0.2 & 0.6 & 0.4 & 0.2 & 0.1 & 1.6 \\
\hline \multicolumn{10}{|c|}{ Age-specific mortality rate per 100,000} \\
\hline World & 7.3 & 4.5 & 10.0 & 35.3 & 209.6 & 694.5 & $1,264.5$ & $2,095.6$ & 128.2 \\
\hline AfrD & 7.6 & 2.9 & 13.5 & 54.6 & 214.0 & 697.7 & $1,607.5$ & $3,446.7$ & 83.9 \\
\hline AfrE & 9.0 & 3.3 & 20.6 & 78.4 & 258.1 & 824.4 & $1,866.5$ & $4,078.5$ & 93.5 \\
\hline AmrA & 3.0 & 2.8 & 7.2 & 28.4 & 190.0 & 689.9 & $1,353.3$ & $2,286.9$ & 213.1 \\
\hline AmrB & 7.4 & 5.4 & 9.6 & 27.1 & 158.5 & 546.5 & $1,139.1$ & $2,145.2$ & 94.5 \\
\hline AmrD & 9.0 & 8.9 & 13.5 & 36.2 & 158.0 & 583.7 & $1,505.9$ & $3,264.8$ & 93.6 \\
\hline EmrB & 8.9 & 6.6 & 9.1 & 22.2 & 137.7 & 461.9 & 904.8 & $\mathrm{I}, 444.8$ & 62.1 \\
\hline EmrD & 12.9 & 8.9 & 11.8 & 39.2 & 134.5 & 385.5 & 704.1 & $1,014.3$ & 54.8 \\
\hline EurA & 3.4 & 3.6 & 7.7 & 31.7 & 230.9 & 762.6 & I,576.8 & $2,7 \mid 1.7$ & 295.6 \\
\hline EurBI & 6.3 & 5.0 & 9.7 & 48.8 & 288.5 & 847.1 & $1,225.6$ & I,657.4 & 183.0 \\
\hline EurB2 & 4.8 & 5.6 & 10.9 & 38.8 & 197.1 & 598.0 & 772.0 & 676.4 & 75.0 \\
\hline EurC & 7.0 & 5.6 & 11.6 & 50.4 & 351.0 & $1,001.0$ & $\mathrm{I}, 453.4$ & $|, 54| . \mid$ & 242.3 \\
\hline SearB & 7.0 & 4.9 & 7.3 & 28.0 & 199.0 & 569.2 & 905.5 & $1,264.7$ & 82.0 \\
\hline SearD & 7.1 & 3.8 & 8.0 & 22.7 & 113.7 & 516.9 & 792.8 & $1,419.2$ & 64.8 \\
\hline WprA & 2.9 & 2.9 & 5.4 & 22.9 & 181.1 & 668.2 & I,387.3 & $2,603.3$ & 270.5 \\
\hline WprBI & 7.5 & 5.1 & 10.6 & 40.3 & 267.2 & 796.0 & $1,372.3$ & I,729.7 & 151.1 \\
\hline WprB2 & 5.9 & 3.8 & 7.6 & 31.3 & I75.I & 431.4 & 784.9 & $2,303.2$ & 68.1 \\
\hline WprB3 & 5.1 & 5.5 & 7.0 & 29.3 & 162.4 & 329.5 & 476.3 & I,404.I & 46.7 \\
\hline
\end{tabular}


Table 5: Estimated global and regional number of total cancer deaths (thousands) and mortality rate (per 100,000) by age, females, 2000

\begin{tabular}{|c|c|c|c|c|c|c|c|c|c|}
\hline \multirow[t]{2}{*}{ Region } & \multicolumn{9}{|c|}{ Age-group } \\
\hline & $0-4$ & $5-14$ & $15-29$ & $30-44$ & $45-59$ & $60-69$ & 70-79 & $80+$ & Total \\
\hline \multicolumn{10}{|c|}{ Number of deaths $(000 s)$} \\
\hline World & 23.4 & 22.0 & 61.4 & 233.8 & 658.2 & 730.6 & 826.4 & 576.2 & $3,132.0$ \\
\hline AfrD & 2.9 & 1.2 & 4.4 & 12.0 & 30.0 & 35.8 & 31.7 & 14.4 & 132.3 \\
\hline AfrE & 2.3 & 1.0 & 5.6 & 12.8 & 31.7 & 40.2 & 35.9 & 14.4 & 144.0 \\
\hline AmrA & 0.3 & 0.5 & 1.6 & 12.6 & 48.4 & 57.8 & 88.7 & 91.1 & 301.1 \\
\hline AmrB & 1.5 & 2.0 & 4.7 & 19.3 & 45.0 & 42.6 & 45.9 & 32.7 & 193.8 \\
\hline AmrD & 0.6 & 0.6 & 1.4 & 4.2 & 9.4 & 8.5 & 9.3 & 5.6 & 39.6 \\
\hline EmrB & 0.4 & 0.8 & 1.8 & 5.0 & 9.2 & 6.6 & 6.2 & 2.9 & 32.9 \\
\hline EmrD & 0.7 & 1.0 & 1.9 & 5.5 & 8.8 & 6.4 & 5.6 & 1.6 & 31.4 \\
\hline EurA & 0.3 & 0.6 & 2.1 & 16.2 & 64.0 & 87.1 & 145.1 & 152.0 & 467.4 \\
\hline EurBI & 0.3 & 0.4 & 1.8 & 7.9 & 22.1 & 27.1 & 29.9 & 14.4 & 104.0 \\
\hline EurB2 & 0.1 & 0.2 & 0.6 & 2.3 & 3.6 & 4.3 & 3.6 & 1.1 & 15.9 \\
\hline EurC & 0.4 & 0.7 & 2.5 & 14.6 & 44.1 & 60.9 & 72.6 & 28.7 & 224.5 \\
\hline SearB & 1.5 & 2.0 & 3.7 & 19.1 & 44.6 & 31.4 & 23.4 & 13.1 & 138.8 \\
\hline SearD & 6.9 & 5.9 & 17.2 & 34.2 & 100.6 & 113.8 & 85.8 & 60.5 & 425.0 \\
\hline WprA & 0.1 & 0.1 & 0.7 & 4.3 & 20.4 & 24.9 & 37.2 & 45.5 & 133.1 \\
\hline WprBI & 4.8 & 4.3 & 9.8 & 58.5 & 165.9 & 175.7 & 198.8 & 91.8 & 709.7 \\
\hline WprB2 & 0.4 & 0.4 & $\mathrm{I} .4$ & 5.0 & 9.9 & 7.2 & 6.4 & 6.2 & 36.9 \\
\hline WprB3 & 0.0 & 0.0 & 0.1 & 0.4 & 0.6 & 0.3 & 0.2 & 0.1 & 1.7 \\
\hline \multicolumn{10}{|c|}{ Age-specific mortality rate per 100,000} \\
\hline World & 8.0 & 3.8 & 8.0 & 37.3 & 163.9 & $4 \mid 4.3$ & 737.9 & I,462.6 & 104.6 \\
\hline AfrD & 10.6 & 2.6 & 9.6 & 44.6 & 206.1 & 641.3 & I,204.5 & $2,286.7$ & 78.9 \\
\hline AfrE & 8.1 & 2.2 & 12.0 & 48.9 & 230.4 & 777.2 & $1,418.8$ & $2,202.6$ & 84.8 \\
\hline AmrA & 2.7 & 2.4 & 5.3 & 34.7 & 166.6 & 484.1 & 862.1 & $1,663.3$ & 193.4 \\
\hline AmrB & 6.6 & 4.4 & 7.7 & 40.9 & 161.3 & 392.0 & 705.7 & I,504.I & 86.9 \\
\hline AmrD & 12.5 & 7.5 & 13.2 & 65.1 & 260.9 & 602.3 & $1,226.6$ & $2,479.1$ & 110.8 \\
\hline EmrB & 5.5 & 4.8 & 9.2 & 42.1 & 151.7 & 280.7 & 474.5 & 909.5 & 49.0 \\
\hline EmrD & 7.4 & 5.9 & 10.1 & 43.8 & 124.7 & 237.8 & 411.1 & 481.1 & 46.1 \\
\hline EurA & 2.9 & 2.6 & 5.4 & 34.6 & 162.9 & 397.0 & 781.7 & I,75I.8 & 224.7 \\
\hline EurBI & 5.0 & 3.4 & 8.3 & 45.3 & 166.0 & 394.8 & 632.1 & $1,134.8$ & 124.4 \\
\hline EurB2 & 2.5 & 4.1 & 9.1 & 44.1 & 150.2 & 326.0 & 463.3 & 491.3 & 61.9 \\
\hline EurC & 6.4 & 4.5 & 9.1 & 52.2 & 192.2 & 417.0 & 661.6 & 851.5 & 172.7 \\
\hline SearB & 7.3 & 5.1 & 6.6 & 45.4 & 187.2 & 329.0 & 491.8 & 991.1 & 70.5 \\
\hline SearD & 8.8 & 4.0 & 9.7 & 27.2 & 136.1 & 383.2 & 580.4 & I,570.4 & 65.1 \\
\hline WprA & 2.0 & 1.7 & 4.7 & 29.0 & 125.6 & 290.5 & 580.5 & $1,509.3$ & 176.2 \\
\hline WprBI & 9.9 & 3.9 & 6.0 & 35.7 & 165.9 & 435.6 & 839.7 & $1,262.3$ & 107.6 \\
\hline WprB2 & 6.0 & 2.9 & 6.8 & 32.1 & 132.6 & 215.4 & 342.3 & $1,113.7$ & 51.5 \\
\hline WprB3 & 3.5 & 3.0 & 9.5 & 68.4 & 184.5 & 233.3 & 294.1 & 960.7 & 51.7 \\
\hline
\end{tabular}

cause groups, including cancers. For regions with insufficient vital registration such as AFRO and SEARO regions, this process significantly increases the mortality envelope for these two broad cause categories. It is possible that these methods result in an overestimate of total cancer deaths in some regions, and continuos efforts are being made to obtain additional data from these regions in order to check the validity of these estimates, and where appropriate, to improve them.
Although cancer is still a fatal disease in many developing countries[2], there is a growing evidence that cancer survival even in developing countries is continuously improving $[3,4]$. The present study suggests that in all regions mortality was much higher in males while incidence were almost the same due to relatively good prognosis of breast and cervix cancers compared to common cancers among males such as liver and lung cancers. Due to the differences in survival by region, incidence-to-mortality rate ratios were higher in developed in which increasing disability among cancer survivors is warranted 
Table 6: Estimated global and regional number of cancer deaths and age-standardised mortality rate by site, males, 2000

\begin{tabular}{|c|c|c|c|c|c|c|c|c|c|c|c|c|c|c|c|c|c|c|}
\hline \multirow[b]{3}{*}{ Site } & \multirow{3}{*}{ World } & \multicolumn{17}{|c|}{ WHO sub region } \\
\hline & & \multicolumn{2}{|c|}{ AFRO } & \multicolumn{3}{|c|}{ AMRO } & \multicolumn{2}{|c|}{ EMRO } & \multicolumn{4}{|c|}{ EURO } & \multicolumn{2}{|c|}{ SEARO } & \multicolumn{4}{|c|}{ WPRO } \\
\hline & & $\mathrm{D}$ & $E$ & $A$ & B & $\mathrm{D}$ & B & $\mathrm{D}$ & $A$ & $\mathrm{BI}$ & B2 & C & B & $\mathrm{D}$ & $A$ & $\mathrm{BI}$ & B2 & B3 \\
\hline \multicolumn{19}{|c|}{ Number of deaths (000s) } \\
\hline $\begin{array}{l}\text { Mouth and } \\
\text { oropharynx } \\
\text { cancers }\end{array}$ & 222.4 & 10.7 & 14.3 & 6.6 & 8.5 & 0.7 & 2.0 & 1.5 & 19.2 & 6.0 & 0.8 & 14.7 & 13.1 & 88.9 & 4.1 & 25.5 & 5.4 & 0.4 \\
\hline $\begin{array}{l}\text { Oesophagus } \\
\text { cancer }\end{array}$ & 273.6 & 4.9 & 13.8 & 11.6 & 10.5 & 0.7 & 1.8 & 0.7 & 21.4 & 3.4 & 1.7 & 10.0 & 3.3 & 41.9 & 9.4 & 135.7 & 2.9 & 0.0 \\
\hline $\begin{array}{l}\text { Stomach } \\
\text { cancer }\end{array}$ & 512.0 & 12.0 & 9.8 & 10.0 & 25.8 & 7.6 & 7.5 & 2.3 & 38.9 & 14.9 & 3.5 & 42.2 & 7.1 & 28.1 & 34.3 & 261.1 & 6.8 & 0.1 \\
\hline $\begin{array}{l}\text { Colon and } \\
\text { rectum } \\
\text { cancers }\end{array}$ & 311.8 & 7.4 & 8.8 & 36.1 & 14.4 & 1.6 & 2.2 & 2.3 & 72.1 & 14.3 & 1.0 & 28.7 & 13.9 & 19.6 & 23.1 & 63.3 & 2.9 & 0.1 \\
\hline Liver cancer & 414.9 & 20.6 & 23.5 & 8.7 & 9.3 & 2.8 & 3.0 & 2.3 & 25.3 & 5.8 & 0.7 & 7.8 & 25.9 & 17.3 & 23.8 & 231.5 & 6.3 & 0.2 \\
\hline $\begin{array}{l}\text { Pancreas } \\
\text { cancer }\end{array}$ & 117.0 & 1.5 & 2.7 & 16.0 & 7.9 & 1.1 & 0.9 & 1.0 & 26.7 & 5.8 & 1.0 & 11.2 & 3.5 & 7.6 & 11.2 & 18.4 & 0.5 & 0.0 \\
\hline $\begin{array}{l}\text { Trachea, } \\
\text { bronchus, } \\
\text { lung cancers }\end{array}$ & 877.3 & 7.0 & 9.4 & 101.4 & 36.1 & 1.6 & 8.7 & 7.1 & 158.0 & 46.6 & 3.8 & 83.6 & 40.8 & 102.7 & 43.2 & 214.6 & 12.4 & 0.3 \\
\hline $\begin{array}{l}\text { Melanoma of } \\
\text { the skin }\end{array}$ & 35.1 & 2.4 & 2.2 & 7.7 & 3.3 & 0.6 & 0.2 & 0.1 & 8.3 & 2.0 & 0.2 & 2.5 & 0.7 & 1.4 & 1.7 & 1.6 & 0.1 & 0.0 \\
\hline Breast cancer & 2.6 & 0.0 & 0.0 & 0.5 & 0.3 & 0.0 & 0.0 & 0.0 & 0.9 & 0.2 & 0.1 & 0.5 & 0.0 & 0.0 & 0.1 & 0.0 & 0.0 & 0.0 \\
\hline $\begin{array}{l}\text { Cervix uteri } \\
\text { cancer }\end{array}$ & 0.0 & 0.0 & 0.0 & 0.0 & 0.0 & 0.0 & 0.0 & 0.0 & 0.0 & 0.0 & 0.0 & 0.0 & 0.0 & 0.0 & 0.0 & 0.0 & 0.0 & 0.0 \\
\hline $\begin{array}{l}\text { Corpus uteri } \\
\text { cancer }\end{array}$ & 0.0 & 0.0 & 0.0 & 0.0 & 0.0 & 0.0 & 0.0 & 0.0 & 0.0 & 0.0 & 0.0 & 0.0 & 0.0 & 0.0 & 0.0 & 0.0 & 0.0 & 0.0 \\
\hline Ovary cancer & 0.0 & 0.0 & 0.0 & 0.0 & 0.0 & 0.0 & 0.0 & 0.0 & 0.0 & 0.0 & 0.0 & 0.0 & 0.0 & 0.0 & 0.0 & 0.0 & 0.0 & 0.0 \\
\hline $\begin{array}{l}\text { Prostate } \\
\text { cancer }\end{array}$ & 263.6 & 26.0 & 19.2 & 39.6 & 30.4 & 5.8 & 2.1 & 1.7 & 70.2 & 9.4 & 0.5 & 13.3 & 8.1 & 19.8 & 10.4 & 6.1 & 0.9 & 0.0 \\
\hline $\begin{array}{l}\text { Bladder } \\
\text { cancer }\end{array}$ & 123.9 & 6.9 & 4.3 & 10.3 & 4.9 & 0.5 & 2.6 & 7.7 & 27.1 & 7.1 & 0.7 & 10.4 & 4.4 & 15.9 & 4.1 & 15.9 & 1.0 & 0.0 \\
\hline $\begin{array}{l}\text { Lymphomas, } \\
\text { multiple } \\
\text { myeloma }\end{array}$ & 168.6 & 15.9 & 13.7 & 23.1 & 9.6 & 2.1 & 4.1 & 3.6 & 27.6 & 5.6 & 0.9 & 5.4 & 9.6 & 26.1 & 7.8 & 11.2 & 2.0 & 0.1 \\
\hline Leukaemia & 145.3 & 5.2 & 6.9 & 14.5 & 9.6 & 2.0 & 2.3 & 3.7 & 20.0 & 5.4 & 0.8 & 7.8 & 7.6 & 21.0 & 4.9 & 31.9 & 1.7 & 0.1 \\
\hline $\begin{array}{l}\text { Other } \\
\text { malignant } \\
\text { neoplasms }\end{array}$ & 425.2 & 18.7 & 25.6 & 39.0 & 36.5 & 6.0 & 7.5 & 4.2 & 79.1 & 24.0 & 3.2 & 41.3 & 16.6 & 60.5 & 20.4 & 37.4 & 5.0 & 0.2 \\
\hline \multicolumn{19}{|c|}{ Age-standardized mortality rate per 100,000} \\
\hline $\begin{array}{l}\text { Mouth and } \\
\text { oropharynx } \\
\text { cancers }\end{array}$ & 7.3 & 11.7 & 16.6 & 2.8 & 4.5 & 2.8 & 4.0 & 3.1 & 5.5 & 5.9 & 4.0 & 9.0 & 8.0 & 16.7 & 2.8 & 3.4 & 10.7 & 18.4 \\
\hline $\begin{array}{l}\text { Oesophagus } \\
\text { cancer }\end{array}$ & 9.0 & 5.5 & 16.4 & 4.8 & 5.6 & 3.0 & 3.6 & 1.6 & 5.6 & 3.2 & 9.0 & 5.9 & 2.1 & 7.9 & 6.1 & 18.3 & 6.1 & 1.9 \\
\hline $\begin{array}{l}\text { Stomach } \\
\text { cancer }\end{array}$ & 16.8 & 13.5 & 11.7 & 3.9 & 13.7 & 31.5 & 15.1 & 4.9 & 9.3 & 14.2 & 18.7 & 25.1 & 4.5 & 5.3 & 21.5 & 35.5 & 14.2 & 3.3 \\
\hline $\begin{array}{l}\text { Colon and } \\
\text { rectum } \\
\text { cancers }\end{array}$ & 10.2 & 8.1 & 11.0 & 13.7 & 7.6 & 6.5 & 4.2 & 4.8 & 17.0 & 13.6 & 5.2 & 17.0 & 8.9 & 3.7 & 14.6 & 8.7 & 6.0 & 4.1 \\
\hline Liver cancer & 13.6 & 20.9 & 25.6 & 3.6 & 4.9 & II.I & 6.2 & 5.0 & 6.2 & 5.6 & 3.6 & 4.6 & 15.9 & 3.1 & 15.7 & 30.5 & 12.2 & 10.1 \\
\hline $\begin{array}{l}\text { Pancreas } \\
\text { cancer }\end{array}$ & 3.8 & 1.6 & 3.4 & 6.3 & 4.2 & 4.7 & 1.8 & 2.2 & 6.6 & 5.6 & 5.3 & 6.7 & 2.2 & 1.5 & 7.1 & 2.5 & 1.0 & 0.2 \\
\hline $\begin{array}{l}\text { Trachea, } \\
\text { bronchus, } \\
\text { lung cancers }\end{array}$ & 28.8 & 7.9 & 11.5 & 40.1 & 19.3 & 6.5 & 17.7 & 15.9 & 39.5 & 44.2 & 19.7 & 48.9 & 26.0 & 19.4 & 26.3 & 29.1 & 25.1 & 13.8 \\
\hline $\begin{array}{l}\text { Melanoma of } \\
\text { the skin }\end{array}$ & 1.2 & 2.7 & 2.6 & 3.2 & 1.7 & 2.5 & 0.4 & 0.2 & 2.2 & 2.0 & 1.1 & 1.6 & 0.5 & 0.3 & 1.1 & 0.2 & 0.2 & 2.4 \\
\hline
\end{tabular}


Table 6: Estimated global and regional number of cancer deaths and age-standardised mortality rate by site, males, 2000 (Continued)

\begin{tabular}{|c|c|c|c|c|c|c|c|c|c|c|c|c|c|c|c|c|c|c|}
\hline Breast cancer & 0.1 & 0.0 & 0.0 & 0.2 & 0.2 & 0.1 & 0.0 & 0.0 & 0.2 & 0.2 & 0.3 & 0.3 & 0.0 & 0.0 & 0.1 & 0.0 & 0.0 & 0.0 \\
\hline $\begin{array}{l}\text { Cervix uteri } \\
\text { cancer }\end{array}$ & 0.0 & 0.0 & 0.0 & 0.0 & 0.0 & 0.0 & 0.0 & 0.0 & 0.0 & 0.0 & 0.0 & 0.0 & 0.0 & 0.0 & 0.0 & 0.0 & 0.0 & 0.0 \\
\hline $\begin{array}{l}\text { Corpus uteri } \\
\text { cancer }\end{array}$ & 0.0 & 0.0 & 0.0 & 0.0 & 0.0 & 0.0 & 0.0 & 0.0 & 0.0 & 0.0 & 0.0 & 0.0 & 0.0 & 0.0 & 0.0 & 0.0 & 0.0 & 0.0 \\
\hline Ovary cancer & 0.0 & 0.0 & 0.0 & 0.0 & 0.0 & 0.0 & 0.0 & 0.0 & 0.0 & 0.0 & 0.0 & 0.0 & 0.0 & 0.0 & 0.0 & 0.0 & 0.0 & 0.0 \\
\hline $\begin{array}{l}\text { Prostate } \\
\text { cancer }\end{array}$ & 8.7 & 33.3 & 27.9 & 12.7 & 16.2 & 24.9 & 4.5 & 4.3 & 14.3 & 8.8 & 2.9 & 7.8 & 5.6 & 4.1 & 5.7 & 0.9 & 1.9 & 1.5 \\
\hline $\begin{array}{l}\text { Bladder } \\
\text { cancer }\end{array}$ & 4.1 & 8.4 & 5.8 & 3.6 & 2.6 & 2.2 & 5.6 & 17.7 & 6.0 & 6.7 & 4.0 & 6.1 & 2.9 & 3.1 & 2.3 & 2.3 & 2.3 & 0.8 \\
\hline $\begin{array}{l}\text { Lymphomas, } \\
\text { multiple } \\
\text { myeloma }\end{array}$ & 5.5 & I5.I & 13.5 & 9.2 & 4.9 & 8.3 & 7.3 & 6.3 & 7.1 & 5.7 & 4.4 & 3.5 & 5.8 & 4.6 & 5.1 & 1.5 & 3.6 & 5.5 \\
\hline Leukaemia & 4.8 & 4.9 & 6.7 & 5.9 & 4.7 & 6.5 & 3.7 & 5.9 & 5.4 & 5.7 & 3.4 & 5.3 & 4.2 & 3.3 & 3.9 & 4.6 & 2.6 & 2.6 \\
\hline $\begin{array}{l}\text { Other } \\
\text { malignant } \\
\text { neoplasms }\end{array}$ & 14.0 & 20.2 & 31.0 & 16.6 & 18.9 & 23.0 & 14.6 & 8.4 & 21.4 & 23.6 & 16.0 & 26.1 & 10.2 & 11.3 & 13.6 & 5.2 & 10.3 & 8.5 \\
\hline
\end{tabular}

Table 7: Estimated global and regional number of cancer deaths and age-standardised mortality rate by site, females, 2000

\begin{tabular}{|c|c|c|c|c|c|c|c|c|c|c|c|c|c|c|c|c|c|c|}
\hline \multirow[b]{3}{*}{ Site } & \multirow{3}{*}{ World } & \multicolumn{17}{|c|}{ WHO sub region } \\
\hline & & \multicolumn{2}{|c|}{ AFRO } & \multicolumn{3}{|c|}{ AMRO } & \multicolumn{2}{|c|}{ EMRO } & \multicolumn{4}{|c|}{ EURO } & \multicolumn{2}{|c|}{ SEARO } & \multicolumn{4}{|c|}{ WPRO } \\
\hline & & $\mathrm{D}$ & $E$ & A & B & $\mathrm{D}$ & B & $\mathrm{D}$ & A & $\mathrm{BI}$ & B2 & $\mathrm{C}$ & B & $\mathrm{D}$ & A & $\mathrm{BI}$ & B2 & B3 \\
\hline \multicolumn{19}{|c|}{ Number of deaths $(000 \mathrm{~s})$} \\
\hline $\begin{array}{l}\text { Mouth and } \\
\text { oropharynx } \\
\text { cancers }\end{array}$ & 97.2 & 5.3 & 7.1 & 3.3 & 2.7 & 0.5 & I.I & 0.6 & 5.7 & 1.5 & 0.3 & 2.9 & 5.5 & 42.7 & 1.7 & 13.6 & 2.6 & 0.2 \\
\hline $\begin{array}{l}\text { Oesophagus } \\
\text { cancer }\end{array}$ & 157.6 & 4.0 & 6.8 & 3.7 & 3.8 & 0.3 & 1.5 & 0.5 & 7.8 & 1.0 & 1.3 & 3.5 & 2.3 & 33.6 & 2.0 & 83.6 & 2.0 & 0.0 \\
\hline $\begin{array}{l}\text { Stomach } \\
\text { cancer }\end{array}$ & 324.2 & 8.9 & 7.2 & 7.0 & 16.5 & 7.1 & 3.7 & 1.6 & 27.9 & 8.6 & 2.2 & 30.6 & 5.5 & 16.1 & 19.0 & 157.7 & 4.6 & 0.1 \\
\hline $\begin{array}{l}\text { Colon and } \\
\text { rectum } \\
\text { cancers }\end{array}$ & 295.5 & 5.6 & 5.3 & 37.2 & 16.1 & 2.1 & 2.2 & 1.7 & 69.7 & 11.8 & 1.1 & $\begin{array}{c}33.6 \\
1\end{array}$ & 3.6 & $|4.0|$ & 9.4 & 59.72 & 2.2 & 0.1 \\
\hline Liver cancer & 191.0 & 11.0 & 9.8 & 5.3 & 9.1 & 3.2 & 1.2 & I.I & 13.3 & 3.8 & 0.5 & 5.6 & 10.6 & 10.4 & 10.5 & 93.5 & 2.2 & 0.2 \\
\hline $\begin{array}{l}\text { Pancreas } \\
\text { cancer }\end{array}$ & 105.6 & 1.5 & 2.5 & 16.9 & 8.7 & 1.2 & 0.7 & 0.6 & 26.9 & 4.6 & 0.7 & 9.8 & 3.3 & 5.2 & 9.4 & 13.0 & 0.5 & 0.0 \\
\hline $\begin{array}{l}\text { Trachea, } \\
\text { bronchus, } \\
\text { lung cancers }\end{array}$ & 323.8 & 2.9 & 4.5 & 70.5 & 14.9 & 0.9 & 2.5 & 2.3 & 49.2 & 9.8 & 1.1 & 16.2 & 7.5 & 23.2 & 16.9 & 97.6 & 3.7 & 0.2 \\
\hline $\begin{array}{l}\text { Melanoma of } \\
\text { the skin }\end{array}$ & 30.1 & 2.4 & 3.0 & 4.3 & 2.7 & 0.6 & 0.2 & 0.1 & 7.2 & 1.9 & 0.2 & 3.0 & 1.0 & 1.0 & 1.1 & 1.2 & 0.1 & 0.0 \\
\hline Breast cancer & 466.3 & 18.6 & 23.0 & 53.3 & 30.7 & 4.0 & 5.1 & 7.0 & 91.8 & 18.5 & 2.5 & 39.4 & 29.0 & 67.0 & 12.4 & 57.5 & 6.3 & 0.2 \\
\hline $\begin{array}{l}\text { Cervix uteri } \\
\text { cancer }\end{array}$ & 259.6 & 24.1 & 36.8 & 5.3 & 19.3 & 4.6 & 3.5 & 2.6 & 8.3 & 6.3 & 1.0 & 11.7 & 15.4 & 86.2 & 2.6 & 26.1 & 5.4 & 0.3 \\
\hline $\begin{array}{l}\text { Corpus uteri } \\
\text { cancer }\end{array}$ & 72.9 & 1.4 & 1.7 & 8.2 & 10.4 & 3.5 & 0.4 & 0.3 & 16.0 & 4.3 & 0.8 & 11.0 & 4.0 & 3.2 & 3.3 & 4.1 & 0.2 & 0.0 \\
\hline Ovary cancer & 128.6 & 3.9 & 6.7 & 15.5 & 7.0 & 1.1 & 0.9 & 0.9 & 25.7 & 5.7 & 0.6 & 12.9 & 8.7 & 18.5 & 4.9 & 13.8 & 1.6 & 0.1 \\
\hline $\begin{array}{l}\text { Prostate } \\
\text { cancer }\end{array}$ & 0.0 & 0.0 & 0.0 & 0.0 & 0.0 & 0.0 & 0.0 & 0.0 & 0.0 & 0.0 & 0.0 & 0.0 & 0.0 & 0.0 & 0.0 & 0.0 & 0.0 & 0.0 \\
\hline $\begin{array}{l}\text { Bladder } \\
\text { cancer }\end{array}$ & 53.5 & 2.3 & 2.0 & 4.9 & 2.1 & 0.3 & 0.5 & 2.0 & 10.5 & 1.7 & 0.2 & 2.8 & 1.4 & 14.2 & 1.9 & 6.3 & 0.6 & 0.0 \\
\hline $\begin{array}{l}\text { Lymphomas, } \\
\text { multiple } \\
\text { myeloma }\end{array}$ & 163.7 & 6.6 & 5.2 & 21.7 & 8.0 & 1.6 & 2.8 & 2.4 & 27.4 & 4.3 & 0.5 & 4.8 & 6.1 & 54.8 & 6.2 & 9.4 & 1.8 & 0.1 \\
\hline Leukaemia & 114.7 & 4.4 & 4.9 & 12.0 & 8.4 & 1.9 & 1.7 & 2.5 & 17.2 & 3.8 & 0.7 & 7.2 & 7.2 & 14.7 & 3.6 & 23.1 & 1.4 & 0.1 \\
\hline
\end{tabular}


Table 7: Estimated global and regional number of cancer deaths and age-standardised mortality rate by site, females, 2000 (Continued)

\begin{tabular}{|c|c|c|c|c|c|c|c|c|c|c|c|c|c|c|c|c|c|c|}
\hline $\begin{array}{l}\text { Other } \\
\text { malignant } \\
\text { neoplasms }\end{array}$ & 344.5 & 29.4 & 14.8 & 32.0 & 33.2 & 6.9 & 5.1 & 5.1 & 63.0 & 16.4 & 2.4 & 29.6 & 15.5 & 19.9 & 18.3 & 49.5 & 3.4 & 0.2 \\
\hline \multicolumn{19}{|c|}{ Age-standardized mortality rate per 100,000} \\
\hline $\begin{array}{l}\text { Mouth and } \\
\text { oropharynx } \\
\text { cancers }\end{array}$ & 3.2 & 6.6 & 8.8 & 1.3 & 1.5 & 2.2 & 2.5 & 1.3 & 1.4 & 1.4 & 1.4 & 1.3 & 3.7 & 9.9 & 1.0 & 2.0 & 4.9 & 10.8 \\
\hline $\begin{array}{l}\text { Oesophagus } \\
\text { cancer }\end{array}$ & 5.3 & 5.0 & 8.8 & 1.3 & 2.1 & 1.4 & 3.8 & I.I & 1.6 & 0.9 & 6.1 & 1.4 & 1.6 & 7.7 & 1.2 & 12.7 & 3.8 & 1.7 \\
\hline $\begin{array}{l}\text { Stomach } \\
\text { cancer }\end{array}$ & 10.8 & 11.3 & 9.2 & 2.5 & 9.1 & 32.2 & 9.0 & 3.6 & 5.8 & 7.9 & 10.6 & 13.0 & 3.7 & 3.8 & 11.5 & 24.1 & 8.7 & 2.6 \\
\hline $\begin{array}{l}\text { Colon and } \\
\text { rectum } \\
\text { cancers }\end{array}$ & 9.9 & 7.0 & 7.0 & 13.0 & 8.9 & 9.4 & 5.6 & 3.7 & 14.4 & 10.7 & 5.3 & 13.9 & 9.3 & 3.3 & 11.6 & 9.1 & 4.2 & 2.6 \\
\hline Liver cancer & 6.4 & 12.4 & 11.5 & 2.0 & 5.0 & 13.9 & 3.0 & 2.4 & 2.9 & 3.4 & 2.2 & 2.4 & 7.1 & 2.1 & 6.2 & 13.9 & 3.9 & 6.9 \\
\hline $\begin{array}{l}\text { Pancreas } \\
\text { cancer }\end{array}$ & 3.5 & 1.7 & 3.4 & 6.1 & 4.8 & 5.6 & 1.7 & 1.5 & 5.7 & 4.1 & 3.4 & 4.1 & 2.3 & 1.2 & 5.5 & 2.0 & 0.9 & 0.3 \\
\hline $\begin{array}{l}\text { Trachea, } \\
\text { bronchus, } \\
\text { lung cancers }\end{array}$ & 10.8 & 3.2 & 5.3 & 27.4 & 8.0 & 4.0 & 6.0 & 5.2 & 11.6 & 9.0 & 5.1 & 6.9 & 4.9 & 4.9 & 10.1 & 14.5 & 6.8 & 7.7 \\
\hline $\begin{array}{l}\text { Melanoma of } \\
\text { the skin }\end{array}$ & 1.0 & 3.1 & 4.1 & 1.7 & 1.5 & 2.6 & 0.4 & 0.3 & 1.7 & 1.8 & 1.0 & 1.5 & 0.7 & 0.2 & 0.7 & 0.2 & 0.1 & 1.5 \\
\hline Breast cancer & 15.6 & 21.4 & 27.7 & 21.1 & 16.0 & 16.1 & 12.2 & 14.4 & 22.8 & 17.4 & 12.0 & 18.7 & 17.6 & 14.5 & 9.4 & 8.1 & 11.3 & 12.0 \\
\hline $\begin{array}{l}\text { Cervix uteri } \\
\text { cancer }\end{array}$ & 8.7 & 27.4 & 43.8 & 2.4 & 9.8 & 18.7 & 8.7 & 5.5 & 2.3 & 6.1 & 4.7 & 5.9 & 9.3 & 18.0 & 2.0 & 3.9 & 9.7 & 15.5 \\
\hline $\begin{array}{l}\text { Corpus uteri } \\
\text { cancer }\end{array}$ & 2.4 & 1.9 & 2.3 & 3.1 & 5.5 & 1.5 & 1.0 & 0.8 & 3.5 & 3.9 & 3.8 & 4.7 & 2.6 & 0.8 & 2.1 & 0.6 & 0.4 & 1.2 \\
\hline Ovary cancer & 4.3 & 4.3 & 8.1 & 6.1 & 3.7 & 4.4 & 2.2 & 1.8 & 6.3 & 5.3 & 3.0 & 6.0 & 5.3 & 4.1 & 3.5 & 2.0 & 2.9 & 3.2 \\
\hline $\begin{array}{l}\text { Prostate } \\
\text { cancer }\end{array}$ & 0.0 & 0.0 & 0.0 & 0.0 & 0.0 & 0.0 & 0.0 & 0.0 & 0.0 & 0.0 & 0.0 & 0.0 & 0.0 & 0.0 & 0.0 & 0.0 & 0.0 & 0.0 \\
\hline $\begin{array}{l}\text { Bladder } \\
\text { cancer }\end{array}$ & 1.8 & 2.9 & 2.6 & 1.6 & 1.2 & 1.2 & 1.4 & 4.6 & 2.0 & 1.5 & 0.9 & I.I & 1.1 & 3.1 & 1.0 & 1.0 & 1.1 & 0.2 \\
\hline $\begin{array}{l}\text { Lymphomas, } \\
\text { multiple } \\
\text { myeloma }\end{array}$ & 5.5 & 6.7 & 5.6 & 7.9 & 4.2 & 6.5 & 6.3 & 4.8 & 6.1 & 4.1 & 2.4 & 2.3 & 3.9 & 11.7 & 3.8 & 1.4 & 3.0 & 3.1 \\
\hline Leukaemia & 3.8 & 4.7 & 5.1 & 4.6 & 4.1 & 6.5 & 3.1 & 4.3 & 4.1 & 3.8 & 2.8 & 3.8 & 4.1 & 2.6 & 2.7 & 3.6 & 2.1 & 2.2 \\
\hline $\begin{array}{l}\text { Other } \\
\text { malignant } \\
\text { neoplasms }\end{array}$ & 11.5 & 34.3 & 18.0 & 12.5 & 17.6 & 28.6 & 12.5 & 10.9 & 14.6 & 15.3 & 11.0 & 14.1 & 10.1 & 4.9 & 11.0 & 7.7 & 6.3 & 8.2 \\
\hline
\end{tabular}

Table 8: Ranking of the global cancer deaths by site, 2000

\begin{tabular}{|c|c|c|}
\hline site & Number of death (000s) & Proportion of total (\%) \\
\hline \multicolumn{3}{|l|}{ Both sexes } \\
\hline Trachea, bronchus, and lung & 1211.5 & 17.2 \\
\hline Stomach & 835.1 & 11.9 \\
\hline Liver & 611.4 & 8.7 \\
\hline Colon and rectum & 608.0 & 8.6 \\
\hline Breast & 473.8 & 6.7 \\
\hline Oesophagus & 430.4 & 6.1 \\
\hline Lymphomas and myeloma & 329.9 & 4.7 \\
\hline Mouth and oropharynx & 320.0 & 4.5 \\
\hline Prostate & 264.0 & 3.8 \\
\hline Leukaemia & 263.8 & 3.8 \\
\hline Cervix uteri & 254.2 & 3.6 \\
\hline Pancreas & 222.3 & 3.2 \\
\hline Bladder & 179.4 & 2.6 \\
\hline Ovary & 130.1 & 1.8 \\
\hline
\end{tabular}


Table 8: Ranking of the global cancer deaths by site, 2000 (Continued)

\begin{tabular}{|c|c|c|}
\hline Corpus uteri & 73.5 & 1.0 \\
\hline Melanoma of the skin & 65.3 & 0.9 \\
\hline \multicolumn{3}{|l|}{ Males } \\
\hline Trachea, bronchus, and lung & 877.2 & 22.5 \\
\hline Stomach & 511.3 & 13.1 \\
\hline Liver & 419.4 & 10.7 \\
\hline Colon and rectum & 312.3 & 8.0 \\
\hline Oesophagus & 272.8 & 7.0 \\
\hline Prostate & 264.0 & 6.8 \\
\hline Mouth and oropharynx & 222.0 & 5.7 \\
\hline Lymphomas and myeloma & 169.6 & 4.3 \\
\hline Leukaemia & 145.4 & 3.7 \\
\hline Bladder & 126.1 & 3.2 \\
\hline Pancreas & 116.8 & 3.0 \\
\hline Melanoma of the skin & 35.0 & 0.9 \\
\hline \multicolumn{3}{|l|}{ Females } \\
\hline Breast & 471.2 & 15.0 \\
\hline Trachea, bronchus, and lung & 334.2 & 10.7 \\
\hline Stomach & 323.7 & 10.3 \\
\hline Colon and rectum & 295.7 & 9.4 \\
\hline Cervix uteri & 254.2 & 8.1 \\
\hline Liver & 192.1 & 6.1 \\
\hline Lymphomas and myeloma & 160.2 & 5.1 \\
\hline Oesophagus & 157.6 & 5.0 \\
\hline Ovary & 130.1 & 4.2 \\
\hline Leukaemia & 118.4 & 3.8 \\
\hline Pancreas & 105.5 & 3.4 \\
\hline Mouth and oropharynx & 98.0 & 3.1 \\
\hline Corpus uteri & 73.5 & 2.3 \\
\hline Bladder & 53.4 & 1.7 \\
\hline Melanoma of the skin & 30.3 & 1.0 \\
\hline
\end{tabular}

Table 9: Ranking of selected regional cancer deaths by site, 2000

\begin{tabular}{|c|c|c|c|c|c|c|c|c|}
\hline \multicolumn{9}{|c|}{ WHO sub region } \\
\hline \multicolumn{3}{|c|}{ AfrE (high child and adult mortality) } & \multicolumn{3}{|c|}{ EurA (very low child and adult mortality) } & \multicolumn{3}{|c|}{ SearB (low child and adult mortality) } \\
\hline site & $\begin{array}{l}\text { Number of } \\
\text { death }(000 \mathrm{~s})\end{array}$ & $\begin{array}{l}\text { Proportion } \\
\text { of total (\%) }\end{array}$ & site & $\begin{array}{l}\text { Number of } \\
\text { death }(000 \mathrm{~s})\end{array}$ & $\begin{array}{c}\text { Proportion } \\
\text { death of } \\
\text { total }(\%)\end{array}$ & site & $\begin{array}{l}\text { Number of } \\
\text { death }(000 \mathrm{~s})\end{array}$ & $\begin{array}{c}\text { Proportion } \\
\text { death of } \\
\text { total (\%) }\end{array}$ \\
\hline \multicolumn{9}{|l|}{ Both sexes } \\
\hline Cervix uteri & 37.3 & 12.4 & $\begin{array}{l}\text { Trachea, bron- } \\
\text { chus, and lung }\end{array}$ & 207.1 & 19.5 & $\begin{array}{l}\text { Trachea, bron- } \\
\text { chus, and lung }\end{array}$ & 48.3 & 16.1 \\
\hline Liver & 35.3 & 11.7 & Colon and rectum & $14 \mid .6$ & 13.3 & Liver & 39.4 & 13.1 \\
\hline Breast & 23.8 & 7.9 & Breast & 92.7 & 8.7 & Breast & 29.4 & 9.8 \\
\hline $\begin{array}{l}\text { Mouth and } \\
\text { oropharynx }\end{array}$ & 21.6 & 7.2 & Prostate & 70.1 & 6.6 & $\begin{array}{l}\text { Colon and } \\
\text { rectum }\end{array}$ & 28.5 & 9.5 \\
\hline Oesophagus & 20.7 & 6.9 & Stomach & 66.7 & 6.3 & $\begin{array}{l}\text { Mouth and } \\
\text { oropharynx }\end{array}$ & 19.4 & 6.5 \\
\hline $\begin{array}{l}\text { Lymphomas and } \\
\text { myeloma }\end{array}$ & 19.2 & 6.4 & $\begin{array}{l}\text { Lymphomas and } \\
\text { myeloma }\end{array}$ & 55.0 & 5.2 & $\begin{array}{l}\text { Lymphomas and } \\
\text { myeloma }\end{array}$ & 16.2 & 5.4 \\
\hline Prostate & 19.2 & 6.4 & Pancreas & 53.6 & 5.0 & Cervix uteri & 15.6 & 5.2 \\
\hline Stomach & 17.1 & 5.7 & Liver & 38.5 & 3.6 & Leukaemia & 15.1 & 5.0 \\
\hline $\begin{array}{l}\text { Colon and } \\
\text { rectum }\end{array}$ & 14.3 & 4.8 & Bladder & 37.6 & 3.5 & Stomach & 13.1 & 4.3 \\
\hline
\end{tabular}


Table 9: Ranking of selected regional cancer deaths by site, 2000 (Continued)

\begin{tabular}{|c|c|c|c|c|c|c|c|c|}
\hline $\begin{array}{l}\text { Trachea, bron- } \\
\text { chus, and lung }\end{array}$ & 14.0 & 4.6 & Leukaemia & 37.2 & 3.5 & Ovary & 8.8 & 2.9 \\
\hline Leukaemia & 12.4 & 4.1 & Oesophagus & 29.2 & 2.7 & Prostate & 8.5 & 2.8 \\
\hline Ovary & 6.9 & 2.3 & Ovary & 25.7 & 2.4 & Pancreas & 7.0 & 2.3 \\
\hline Bladder & 6.3 & 2.1 & $\begin{array}{l}\text { Mouth and } \\
\text { oropharynx }\end{array}$ & 24.9 & 2.3 & Bladder & 6.2 & 2.1 \\
\hline $\begin{array}{l}\text { Melanoma of } \\
\text { the skin }\end{array}$ & 5.3 & 1.8 & Corpus uteri & 16.0 & 1.5 & Oesophagus & 5.9 & 2.0 \\
\hline Pancreas & 5.3 & 1.8 & $\begin{array}{c}\text { Melanoma of the } \\
\text { skin }\end{array}$ & 15.5 & 1.5 & Corpus uteri & 4.1 & 1.4 \\
\hline Corpus uteri & 1.7 & 0.6 & Cervix uteri & 8.3 & 0.8 & $\begin{array}{l}\text { Melanoma of } \\
\text { the skin }\end{array}$ & 1.8 & 0.6 \\
\hline \multicolumn{9}{|l|}{ Males } \\
\hline Liver & 24.9 & 15.9 & $\begin{array}{l}\text { Trachea, bron- } \\
\text { chus, and lung }\end{array}$ & 158.0 & 26.6 & $\begin{array}{l}\text { Trachea, bron- } \\
\text { chus, and lung }\end{array}$ & 40.8 & 25.2 \\
\hline Prostate & 19.2 & 12.2 & Colon and rectum & 72.0 & 12.1 & Liver & 28.4 & 17.6 \\
\hline $\begin{array}{l}\text { Mouth and } \\
\text { oropharynx }\end{array}$ & 14.4 & 9.2 & Prostate & 70.1 & 11.8 & $\begin{array}{l}\text { Colon and } \\
\text { rectum }\end{array}$ & 14.6 & 9.1 \\
\hline $\begin{array}{l}\text { Lymphomas and } \\
\text { myeloma }\end{array}$ & 14.0 & 8.9 & Stomach & 38.8 & 6.5 & $\begin{array}{l}\text { Mouth and } \\
\text { oropharynx }\end{array}$ & 13.8 & 8.5 \\
\hline Oesophagus & 13.8 & 8.8 & $\begin{array}{l}\text { Lymphomas and } \\
\text { myeloma }\end{array}$ & 27.6 & 4.6 & $\begin{array}{l}\text { Lymphomas and } \\
\text { myeloma }\end{array}$ & 10.0 & 6.2 \\
\hline Stomach & 9.9 & 6.3 & Bladder & 27.1 & 4.6 & Prostate & 8.5 & 5.3 \\
\hline $\begin{array}{l}\text { Trachea, bron- } \\
\text { chus, and lung }\end{array}$ & 9.4 & 6.0 & Pancreas & 26.7 & 4.5 & Leukaemia & 7.8 & 4.8 \\
\hline $\begin{array}{l}\text { Colon and } \\
\text { rectum }\end{array}$ & 9.0 & 5.7 & Liver & 25.3 & 4.3 & Stomach & 7.5 & 4.6 \\
\hline Leukaemia & 7.3 & 4.7 & Oesophagus & 21.4 & 3.6 & Bladder & 4.7 & 2.9 \\
\hline Bladder & 4.3 & 2.7 & Leukaemia & 20.0 & 3.4 & Pancreas & 3.6 & 2.2 \\
\hline Pancreas & 2.8 & 1.8 & $\begin{array}{l}\text { Mouth and } \\
\text { oropharynx }\end{array}$ & 19.2 & 3.2 & Oesophagus & 3.6 & 2.2 \\
\hline $\begin{array}{l}\text { Melanoma of } \\
\text { the skin }\end{array}$ & 2.3 & 1.4 & $\begin{array}{l}\text { Melanoma of the } \\
\text { skin }\end{array}$ & 8.3 & 1.4 & $\begin{array}{l}\text { Melanoma of } \\
\text { the skin }\end{array}$ & 0.7 & 0.5 \\
\hline \multicolumn{9}{|l|}{ Females } \\
\hline Cervix uteri & 37.3 & 25.9 & Breast & 91.8 & 19.6 & Breast & 29.3 & 21.1 \\
\hline Breast & 23.8 & 16.5 & Colon and rectum & 69.6 & 14.9 & Cervix uteri & 15.6 & 11.3 \\
\hline Liver & 10.3 & 7.2 & $\begin{array}{l}\text { Trachea, bron- } \\
\text { chus, and lung }\end{array}$ & 49.2 & 10.5 & $\begin{array}{l}\text { Colon and } \\
\text { rectum }\end{array}$ & 13.9 & 10.0 \\
\hline Stomach & 7.2 & 5.0 & Stomach & 27.8 & 6.0 & Liver & 11.0 & 7.9 \\
\hline $\begin{array}{l}\text { Mouth and } \\
\text { oropharynx }\end{array}$ & 7.2 & 5.0 & $\begin{array}{l}\text { Lymphomas and } \\
\text { myeloma }\end{array}$ & 27.4 & 5.9 & Ovary & 8.8 & 6.3 \\
\hline Ovary & 6.9 & 4.8 & Pancreas & 26.9 & 5.8 & $\begin{array}{l}\text { Trachea, bron- } \\
\text { chus, and lung }\end{array}$ & 7.5 & 5.4 \\
\hline Oesophagus & 6.8 & 4.8 & Ovary & 25.7 & 5.5 & Leukaemia & 7.2 & 5.2 \\
\hline $\begin{array}{l}\text { Colon and } \\
\text { rectum }\end{array}$ & 5.3 & 3.7 & Leukaemia & 17.2 & 3.7 & $\begin{array}{l}\text { Lymphomas and } \\
\text { myeloma }\end{array}$ & 6.2 & 4.5 \\
\hline $\begin{array}{l}\text { Lymphomas and } \\
\text { myeloma }\end{array}$ & 5.3 & 3.7 & Corpus uteri & 16.0 & 3.4 & $\begin{array}{l}\text { Mouth and } \\
\text { oropharynx }\end{array}$ & 5.6 & 4.0 \\
\hline Leukaemia & 5.1 & 3.5 & Liver & 13.3 & 2.8 & Stomach & 5.6 & 4.0 \\
\hline $\begin{array}{l}\text { Trachea, bron- } \\
\text { chus, and lung }\end{array}$ & 4.5 & 3.2 & Bladder & 10.5 & 2.2 & Corpus uteri & 4.1 & 2.9 \\
\hline $\begin{array}{l}\text { Melanoma of } \\
\text { the skin }\end{array}$ & 3.0 & 2.1 & Cervix uteri & 8.3 & 1.8 & Pancreas & 3.4 & 2.4 \\
\hline Pancreas & 2.5 & 1.8 & Oesophagus & 7.8 & 1.7 & Oesophagus & 2.4 & 1.7 \\
\hline Bladder & 2.0 & 1.4 & $\begin{array}{c}\text { Melanoma of the } \\
\text { skin }\end{array}$ & 7.2 & 1.5 & Bladder & 1.5 & I.I \\
\hline Corpus uteri & 1.7 & 1.2 & $\begin{array}{l}\text { Mouth and } \\
\text { oropharynx }\end{array}$ & 5.7 & 1.2 & $\begin{array}{l}\text { Melanoma of } \\
\text { the skin }\end{array}$ & 1.0 & 0.7 \\
\hline
\end{tabular}


Table 10: Estimated global site-specific cancer incidence-to-mortality rate ratios by sex

\begin{tabular}{llc}
\hline Site & Male & Female \\
\hline Mouth and oropharynx & 1.20 & 1.57 \\
Oesophagus & 1.05 & 1.05 \\
Stomach & 1.15 & 1.19 \\
Colon and rectum & 1.69 & 1.75 \\
Liver & 1.01 & 1.06 \\
Pancreas & 1.02 & 1.01 \\
Trachea, bronchus, and lung & 1.07 & 1.10 \\
Melanoma of the skin & 3.37 & 2.93 \\
Breast & & 2.19 \\
Cervix uteri & & 1.91 \\
Corpus uteri & & 4.45 \\
Ovary & & 1.70 \\
Prostate & 2.08 & \\
Bladder & 2.21 & 1.01 \\
Lymphomas and multiple myeloma & 1.40 & 1.51 \\
Leukaemia & 1.35 & 1.44 \\
Other malignant neoplasms & 1.49 & 1.63 \\
\hline
\end{tabular}

Table II: Estimated global and regional number of new cases (thousands) and incidence rate (per 100,000) of all cancers by age, both sexes, 2000

\begin{tabular}{|c|c|c|c|c|c|c|c|c|c|}
\hline \multirow[b]{2}{*}{ Region } & \multicolumn{9}{|c|}{ Age group } \\
\hline & $0-4$ & $5-14$ & $15-29$ & $30-44$ & $45-59$ & $60-69$ & 70-79 & $80+$ & Total \\
\hline \multicolumn{10}{|c|}{ Number of new cases $(000$ s) } \\
\hline World & 136.8 & 96.5 & 360.1 & 923.3 & 2459.3 & $264 I .4$ & $25 \mid 4.5$ & 1160.2 & 10292.1 \\
\hline AfrD & 12.5 & 4.6 & 24.7 & 41.9 & 87.6 & 90.8 & 74.5 & 31.9 & 368.6 \\
\hline AfrE & 12.8 & 4.9 & 33.8 & 49.1 & 99.2 & 102.4 & 81.0 & 31.9 & 415.1 \\
\hline AmrA & 3.0 & 3.3 & 22.7 & 110.4 & 306.3 & 308.4 & 404.2 & 176.4 & 1334.8 \\
\hline AmrB & 10.0 & 8.5 & 31.5 & 76.1 & 150.8 & 137.5 & 127.6 & 69.2 & 611.1 \\
\hline AmrD & 2.9 & 2.6 & 7.5 & 15.6 & 28.5 & 23.8 & 22.7 & 11.9 & 115.5 \\
\hline EmrB & 3.5 & 4.3 & 8.8 & 15.7 & 28.4 & 24.0 & 20.5 & 7.9 & 113.2 \\
\hline EmrD & 5.4 & 4.7 & 9.3 & 20.0 & 28.2 & 19.9 & 15.1 & 4.4 & 106.9 \\
\hline EurA & 2.7 & 3.6 & 23.8 & 96.6 & 323.0 & 421.1 & 531.4 & 322.2 & 1724.3 \\
\hline EurBI & 2.1 & 2.2 & 11.0 & 33.8 & 91.6 & 105.0 & 84.4 & 27.1 & 357.2 \\
\hline EurB2 & 0.6 & 1.2 & 3.7 & 8.4 & 11.7 & 14.0 & 8.7 & 1.9 & 50.2 \\
\hline EurC & 2.6 & 3.5 & 18.0 & 60.8 & 172.9 & 222.9 & 180.5 & 46.1 & 707.3 \\
\hline SearB & 8.8 & 7.5 & 18.9 & 65.1 & 138.9 & 103.9 & 67.6 & 26.7 & 437.5 \\
\hline SearD & 39.6 & 24.2 & 85.1 & 111.0 & 277.0 & 317.7 & 208.4 & 110.8 & 1173.9 \\
\hline WprA & 0.9 & 0.9 & 6.8 & 25.7 & 108.2 & 133.0 & 153.0 & 107.7 & 536.1 \\
\hline WprBI & 26.9 & 18.3 & 47.7 & 176.3 & 575.8 & 593.2 & 516.0 & 170.3 & 2124.4 \\
\hline WprB2 & 2.5 & 2.0 & 6.6 & 15.6 & 29.5 & 22.9 & 18.2 & 13.6 & 110.8 \\
\hline WprB3 & 0.1 & 0.1 & 0.4 & 1.2 & 1.9 & 0.9 & 0.5 & 0.2 & 5.2 \\
\hline \multicolumn{10}{|c|}{ Age-specific incidence rate per 100,000} \\
\hline World & 22.6 & 8.1 & 23.0 & 72.4 & 305.6 & 781.8 & 1276.6 & 1830.7 & 170.4 \\
\hline AfrD & 22.9 & 5.2 & 26.8 & 78.9 & 310.4 & 866.4 & 1554.9 & 2834.8 & 110.4 \\
\hline AfrE & 22.3 & 5.2 & 36.3 & 94.3 & 371.8 & 1070.3 & 1812.2 & 2975.0 & 123.0 \\
\hline AmrA & 14.4 & 7.5 & 36.4 & 150.7 & 532.9 & 1356.3 & 2232.0 & 1994.7 & 433.0 \\
\hline AmrB & 21.9 & 9.5 & 25.4 & 82.0 & 279.1 & 676.3 & II03.7 & 1842.4 & 138.2 \\
\hline AmrD & 30.8 & 15.1 & 36.5 & 123.3 & 406.3 & 881.5 & 1623.0 & 2921.2 & 162.0 \\
\hline EmrB & 21.4 & 12.3 & 21.4 & 62.2 & 213.6 & 494.3 & 793.5 & 1206.4 & 81.1 \\
\hline EmrD & 28.9 & 14.2 & 23.8 & 78.2 & 205.5 & 401.7 & 619.9 & 728.7 & 77.5 \\
\hline EurA & 12.4 & 7.5 & 29.6 & 102.1 & $4 \mid 2.5$ & 1008.3 & 1681.0 & 2406.4 & 421.6 \\
\hline
\end{tabular}


Table I I: Estimated global and regional number of new cases (thousands) and incidence rate (per 100,000) of all cancers by age, both sexes, 2000 (Continued)

\begin{tabular}{lccccccccc}
\hline EurBI & 17.0 & 8.5 & 25.3 & 95.7 & 349.8 & 826.0 & 1056.8 & 1355.2 & 215.4 \\
EurB2 & 10.5 & 9.7 & 26.6 & 81.0 & 254.7 & 577.3 & 674.1 & 560.0 & 98.7 \\
EurC & 20.6 & 10.3 & 32.4 & 109.7 & 403.0 & 901.9 & 1125.9 & 1048.5 & 288.5 \\
SearB & 21.4 & 9.3 & 16.8 & 77.4 & 297.8 & 575.8 & 776.5 & 1142.3 & 110.9 \\
SearD & 24.4 & 7.9 & 22.9 & 42.4 & 182.7 & 544.1 & 738.9 & 1521.0 & 87.1 \\
WprA & 10.9 & 5.8 & 22.1 & 86.1 & 334.2 & 808.6 & 1361.9 & 2273.3 & 360.0 \\
WprBI & 26.1 & 7.8 & 14.0 & 52.3 & 278.2 & 728.1 & 1193.0 & 1475.8 & 156.5 \\
WprB2 & 16.6 & 6.3 & 15.8 & 51.7 & 210.3 & 378.2 & 572.8 & 1565.2 & 78.0 \\
WprB3 & 11.7 & 7.7 & 21.6 & 93.3 & 271.0 & 364.6 & 422.9 & 1174.1 & 76.4 \\
\hline
\end{tabular}

Table 12: Estimated global and regional number of new cases (thousands) and incidence rate (per 100,000) of all cancers by age, males, 2000

\begin{tabular}{|c|c|c|c|c|c|c|c|c|c|}
\hline \multirow[b]{2}{*}{ Region } & \multicolumn{9}{|c|}{ Age group } \\
\hline & $0-4$ & $5-14$ & $15-29$ & $30-44$ & $45-59$ & $60-69$ & 70-79 & $80+$ & Total \\
\hline \multicolumn{10}{|c|}{ Number of new cases (000s) } \\
\hline World & 64.5 & 50.9 & 154.0 & 257.7 & 1174.2 & 1528.4 & 1380.8 & 556.5 & 5167.1 \\
\hline AfrD & 5.2 & 2.3 & 10.4 & 13.0 & 37.9 & 45.1 & 39.0 & 17.3 & 170.2 \\
\hline AfrE & 6.6 & 2.8 & 15.3 & 18.3 & 42.5 & 47.2 & 40.0 & 17.2 & 189.9 \\
\hline AmrA & 1.6 & 1.7 & 11.0 & 28.3 & 134.7 & 174.3 & 226.6 & 109.9 & 688.2 \\
\hline $\mathrm{AmrB}$ & 5.3 & 4.4 & 13.9 & 15.7 & 56.1 & 70.5 & 68.2 & 34.6 & 268.7 \\
\hline AmrD & 1.2 & 1.4 & 2.8 & 2.6 & 7.2 & 10.5 & 11.2 & 6.0 & 42.9 \\
\hline EmrB & 2.2 & 2.5 & 3.6 & 3.4 & 13.2 & 14.7 & 13.1 & 4.9 & 57.5 \\
\hline EmrD & 3.4 & 2.7 & 4.3 & 6.3 & 13.3 & 11.5 & 8.5 & 2.7 & 52.8 \\
\hline EurA & 1.5 & 2.0 & 11.5 & 26.4 & 153.8 & 250.2 & 291.4 & 139.6 & 876.3 \\
\hline EurBI & 1.1 & 1.3 & 4.8 & 10.8 & 49.4 & 63.2 & 45.7 & 12.2 & 188.5 \\
\hline EurB2 & 0.4 & 0.7 & 1.6 & 2.2 & 5.3 & 7.9 & 4.3 & 0.7 & 23.1 \\
\hline EurC & 1.4 & 1.8 & 8.1 & 17.5 & 90.3 & 126.3 & 84.3 & 16.0 & 345.6 \\
\hline SearB & 4.3 & 3.3 & 7.0 & 11.8 & 57.2 & 59.7 & 39.5 & 13.0 & 195.8 \\
\hline SearD & 16.2 & 11.8 & 27.0 & 29.8 & 110.2 & 173.8 & 113.7 & 49.1 & 531.6 \\
\hline WprA & 0.5 & 0.5 & 3.0 & 5.8 & 51.0 & 86.0 & 94.3 & 51.3 & 292.3 \\
\hline WprBI & 12.3 & 10.5 & 27.1 & 61.4 & 337.9 & 373.6 & 289.8 & 74.8 & 1187.5 \\
\hline WprB2 & 1.2 & 1.0 & 2.5 & 4.2 & 13.5 & 13.5 & 11.0 & 7.2 & 54.2 \\
\hline WprB3 & 0.1 & 0.1 & 0.1 & 0.2 & 0.7 & 0.5 & 0.3 & 0.1 & 2.0 \\
\hline \multicolumn{10}{|c|}{ Age-specific incidence rate per 100,000} \\
\hline World & 20.8 & 8.3 & 19.2 & 39.8 & 291.3 & 946.2 & 1624.8 & 2320.5 & 169.7 \\
\hline AfrD & 18.7 & 5.2 & 22.6 & 49.5 & 277.5 & 921.0 & 1801.5 & 3472.3 & 102.4 \\
\hline AfrE & 23.1 & 5.9 & 32.8 & 70.5 & 328.9 & 1075.5 & 2067.8 & 4109.5 & 113.2 \\
\hline AmrA & 15.3 & 7.6 & 34.8 & 76.6 & 474.1 & 1614.6 & 2897.4 & 3261.5 & 451.0 \\
\hline $\mathrm{AmrB}$ & 22.6 & 9.6 & 22.4 & 34.4 & 215.1 & 745.8 & 1347.6 & 2190.5 & 122.6 \\
\hline AmrD & 25.6 & 16.0 & 27.0 & 42.0 & 212.4 & $8 \mid 4.8$ & 1742.9 & 3320.7 & 120.7 \\
\hline EmrB & 26.0 & 13.8 & 17.2 & 25.2 & 181.4 & 584.4 & 1018.0 & 1464.3 & 79.6 \\
\hline EmrD & 36.1 & 16.2 & 21.6 & 48.4 & 199.2 & 503.8 & 791.2 & 1024.4 & 75.6 \\
\hline EurA & 13.3 & 8.2 & 27.9 & 55.2 & 394.2 & 1262.9 & 2233.2 & 2960.3 & 435.9 \\
\hline EurBI & 18.5 & 9.5 & 21.8 & 60.5 & 383.2 & 1081.9 & 1403.2 & 1668.1 & 229.1 \\
\hline EurB2 & 13.5 & 10.6 & 22.9 & 43.2 & 238.3 & 710.9 & 851.6 & 678.2 & 91.7 \\
\hline EurC & 21.4 & 10.7 & 28.7 & 63.8 & 451.9 & 1248.4 & 1667.0 & 1551.3 & 299.9 \\
\hline SearB & 20.3 & 8.0 & 12.3 & 28.1 & 250.5 & 702.6 & 1001.8 & |284.| & 99.2 \\
\hline SearD & 19.4 & 7.5 & 14.0 & 21.8 & $|4| .7$ & 606.1 & 847.2 & 1430.6 & 76.5 \\
\hline WprA & 12.8 & 6.7 & 18.9 & 38.6 & 316.3 & 1088.7 & 1953.2 & 2971.8 & 398.4 \\
\hline WprBI & 22.7 & 8.6 & 15.4 & 35.5 & 316.0 & 908.2 & 1480.4 & 1753.4 & 170.2 \\
\hline WprB2 & 15.4 & 6.6 & 11.6 & 28.6 & 207.3 & 500.1 & 835.9 & 2327.7 & 77.2 \\
\hline WprB3 & 13.5 & 9.0 & 10.5 & 27.9 & 214.4 & 400.7 & 515.2 & $14 \mid 2.7$ & 57.5 \\
\hline
\end{tabular}


Table 13: Estimated global and regional number of new cases (thousands) and incidence rate (per 100,000) of all cancers by age, females, 2000

\begin{tabular}{|c|c|c|c|c|c|c|c|c|c|}
\hline \multirow[b]{2}{*}{ Region } & \multicolumn{9}{|c|}{ Age group } \\
\hline & $0-4$ & $5-14$ & $15-29$ & $30-44$ & $45-59$ & $60-69$ & 70-79 & $80+$ & Total \\
\hline \multicolumn{10}{|c|}{ Number of new cases $(000 s)$} \\
\hline World & 72.3 & 45.6 & 206.1 & 665.6 & 1285.1 & 1112.9 & 1133.6 & 603.7 & 5125.0 \\
\hline AfrD & 7.4 & 2.3 & 14.2 & 28.9 & 49.6 & 45.8 & 35.6 & 14.7 & 198.4 \\
\hline AfrE & 6.1 & 2.1 & 18.5 & 30.8 & 56.7 & 55.1 & 41.0 & 14.8 & 225.2 \\
\hline AmrA & 1.4 & 1.6 & 11.7 & 82.1 & 171.6 & 134.2 & 177.7 & 66.5 & 646.7 \\
\hline AmrB & 4.7 & 4.1 & 17.5 & 60.4 & 94.7 & 66.9 & 59.5 & 34.6 & 342.4 \\
\hline AmrD & 1.7 & 1.2 & 4.7 & 13.0 & 21.3 & 13.3 & 11.5 & 5.9 & 72.6 \\
\hline EmrB & 1.3 & 1.8 & 5.2 & 12.4 & 15.2 & 9.3 & 7.4 & 3.0 & 55.6 \\
\hline EmrD & 1.9 & 2.0 & 5.0 & 13.7 & 14.9 & 8.4 & 6.6 & 1.7 & 54.1 \\
\hline EurA & 1.2 & 1.6 & 12.3 & 70.2 & 169.2 & 170.9 & 240.0 & 182.7 & 848.0 \\
\hline EurBI & 0.9 & 0.9 & 6.2 & 23.0 & 42.2 & 41.8 & 38.7 & 14.9 & 168.8 \\
\hline EurB2 & 0.2 & 0.5 & 2.1 & 6.2 & 6.4 & 6.1 & 4.4 & 1.2 & 27.1 \\
\hline EurC & 1.2 & 1.6 & 9.9 & 43.3 & 82.7 & 96.7 & 96.2 & 30.2 & 361.7 \\
\hline SearB & 4.5 & 4.2 & 11.9 & 53.3 & 81.7 & 44.2 & 28.1 & 13.7 & 241.7 \\
\hline SearD & 23.4 & 12.4 & 58.1 & 81.2 & 166.8 & 143.8 & 94.7 & 61.7 & 642.2 \\
\hline WprA & 0.3 & 0.4 & 3.8 & 19.9 & 57.2 & 47.1 & 58.7 & 56.4 & 243.8 \\
\hline WprBI & 14.6 & 7.8 & 20.6 & $1 \mid 4.8$ & 237.8 & 219.6 & 226.2 & 95.5 & 936.9 \\
\hline WprB2 & 1.3 & 0.9 & 4.1 & 11.4 & 15.9 & 9.3 & 7.2 & 6.3 & 56.5 \\
\hline WprB3 & 0.0 & 0.1 & 0.3 & 1.0 & 1.1 & 0.4 & 0.2 & 0.1 & 3.2 \\
\hline \multicolumn{10}{|c|}{ Age-specific incidence rate per 100,000} \\
\hline World & 24.6 & 7.9 & 27.0 & 106.1 & 320.0 & 631.1 & 1012.3 & 1532.5 & $17||$. \\
\hline AfrD & 27.2 & 5.1 & 30.9 & 107.8 & 341.2 & 818.6 & 1352.1 & 2330.7 & 118.3 \\
\hline AfrE & 21.6 & 4.6 & 39.8 & 117.9 & 412.0 & 1065.8 & 1616.9 & 2253.0 & 132.6 \\
\hline AmrA & 13.5 & 7.4 & 38.1 & 226.2 & 590.3 & 1123.0 & 1726.5 & 1214.7 & 415.3 \\
\hline AmrB & 21.2 & 9.3 & 28.4 & 127.8 & 339.0 & 615.8 & 914.1 & 1589.7 & 153.5 \\
\hline AmrD & 36.2 & 14.2 & 46.1 & 200.8 & 588.6 & 942.4 & 1521.8 & 2602.4 & 203.0 \\
\hline EmrB & 16.6 & 10.7 & 25.8 & 104.3 & 252.3 & 397.3 & 571.2 & 937.3 & 82.8 \\
\hline EmrD & 21.5 & 12.1 & 26.1 & 109.2 & 211.4 & 315.0 & 484.3 & 495.4 & 79.3 \\
\hline EurA & 11.5 & 6.8 & 31.5 & 150.3 & 430.7 & 778.6 & 1292.8 & 2105.4 & 407.8 \\
\hline EurBI & 15.4 & 7.4 & 29.0 & 131.6 & 317.4 & 608.6 & 818.5 & 1175.8 & 202.0 \\
\hline EurB2 & 7.5 & 8.8 & 30.4 & 117.2 & 270.0 & 464.6 & 559.4 & 504.5 & 105.5 \\
\hline EurC & 19.7 & 9.9 & 36.1 & 154.6 & 360.5 & 661.9 & 876.5 & 895.0 & 278.3 \\
\hline SearB & 22.5 & 10.8 & 21.3 & 126.4 & 343.1 & 463.0 & 589.7 & 1033.9 & 122.7 \\
\hline SearD & 29.8 & 8.3 & 32.6 & 64.8 & 225.9 & 484.2 & 640.6 & 1601.5 & 98.4 \\
\hline WprA & 9.0 & 4.9 & 25.5 & 134.4 & 351.9 & 550.0 & 916.0 & 1873.2 & 322.7 \\
\hline WprBI & 29.8 & 7.0 & 12.5 & 70.1 & 237.8 & 544.5 & 955.4 & 1312.9 & 142.1 \\
\hline WprB2 & 17.9 & 6.1 & 20.2 & 73.7 & 212.9 & 279.2 & 386.9 & 1138.6 & 78.8 \\
\hline WprB3 & 9.6 & 6.3 & 33.4 & 166.5 & 328.3 & 330.2 & 343.6 & 973.9 & 96.5 \\
\hline
\end{tabular}

[17-19]. It is suggested that data on incidence as well as mortality are necessary to understand the magnitude and trends of cancer problems and to evaluate the interventions against cancer in the context of prevention [1]. When setting priorities, interventions against cancers need to be compared with other health interventions which aims at reducing only morbidity $[5,20]$.

GBD 2000 employs a composite measure of disease burden in terms of disability-adjusted life years (DALYs) which consist of years lived with disability (morbidity) and years of life lost (mortality) [5]. As a part of this exercise, we have estimated both mortality and incidence of cancers by site. We will further estimate the years lived with disability to derive cancer burden in DALYs once weights assigned to disabling conditions are revised based on the estimates from the on-going population-based surveys from more than 70 countries [21].

The presents study also suggests that there is a significant variation in the distribution of cancer mortality and incidence by region depending on age and population struc- 
Table 14: Estimated global and regional number of new cases and age-standardised incidence rate of cancer by site, males, 2000

\begin{tabular}{|c|c|c|c|c|c|c|c|c|c|c|c|c|c|c|c|c|c|c|}
\hline \multirow[b]{3}{*}{ Site } & \multirow{3}{*}{ World } & \multicolumn{17}{|c|}{ WHO sub region } \\
\hline & & \multicolumn{2}{|c|}{ AFRO } & \multicolumn{3}{|c|}{ AMRO } & \multicolumn{2}{|c|}{ EMRO } & \multicolumn{4}{|c|}{ EURO } & \multicolumn{2}{|c|}{ SEARO } & \multicolumn{4}{|c|}{ WPRO } \\
\hline & & $\mathrm{D}$ & $\mathrm{E}$ & A & B & $\mathrm{D}$ & B & $\mathrm{D}$ & $A$ & $\mathrm{BI}$ & B2 & C & B & $\mathrm{D}$ & $A$ & $\mathrm{BI}$ & B2 & B3 \\
\hline \multicolumn{19}{|c|}{ Number of new cases (000s) } \\
\hline $\begin{array}{l}\text { Mouth and } \\
\text { oropharynx } \\
\text { cancers }\end{array}$ & 267.1 & 12.1 & 16.5 & 11.3 & 10.4 & 0.9 & 2.6 & 1.7 & 28.7 & 7.4 & 0.9 & 17.8 & 16.0 & 98.1 & 6.5 & 29.7 & 6.1 & 0.5 \\
\hline $\begin{array}{l}\text { Oesophagus } \\
\text { cancer }\end{array}$ & 286.5 & 5.1 & 14.1 & 12.4 & 11.1 & 0.7 & 1.9 & 0.8 & 22.8 & 3.6 & 1.8 & 10.7 & 3.8 & 42.0 & 10.1 & 142.7 & 3.0 & 0.0 \\
\hline $\begin{array}{l}\text { Stomach } \\
\text { cancer }\end{array}$ & 589.3 & 13.3 & 10.9 & 11.8 & 29.7 & 8.7 & 8.6 & 2.5 & 45.6 & 17.2 & 4.0 & 48.6 & 8.6 & 30.1 & 40.6 & 300.9 & 7.6 & 0.1 \\
\hline $\begin{array}{l}\text { Colon and } \\
\text { rectum } \\
\text { cancers }\end{array}$ & 527.8 & 9.5 & 12.0 & 85.6 & 21.2 & 2.2 & 3.2 & 3.1 & 133.8 & 21.5 & 1.4 & 42.8 & 21.4 & 25.7 & 49.2 & 91.0 & 3.8 & 0.1 \\
\hline Liver cancer & 422.0 & 20.0 & 23.6 & 9.0 & 9.5 & 2.9 & 3.1 & 2.3 & 26.1 & 6.0 & 0.7 & 7.9 & 28.6 & 18.4 & 24.6 & 232.8 & 6.2 & 0.2 \\
\hline $\begin{array}{l}\text { Pancreas } \\
\text { cancer }\end{array}$ & 119.3 & 1.5 & 2.8 & 16.5 & 8.1 & 1.2 & 0.9 & 1.0 & 27.4 & 5.9 & 1.0 & 11.4 & 3.7 & 7.3 & 11.5 & 18.7 & 0.5 & 0.0 \\
\hline $\begin{array}{l}\text { Trachea, } \\
\text { bronchus, } \\
\text { lung cancers }\end{array}$ & 939.9 & 7.4 & 9.8 & 112.1 & 38.5 & 1.7 & 9.3 & 7.4 & 172.0 & 49.5 & 3.9 & 89.3 & 43.1 & 107.8 & 46.9 & 227.8 & 13.1 & 0.3 \\
\hline $\begin{array}{l}\text { Melanoma of } \\
\text { the skin }\end{array}$ & 118.2 & 3.5 & 3.4 & 61.8 & 5.3 & 0.9 & 0.3 & 0.2 & 22.6 & 3.2 & 0.3 & 4.0 & 1.2 & 2.1 & 6.7 & 2.6 & 0.1 & 0.1 \\
\hline $\begin{array}{l}\text { Breast } \\
\text { cancer }\end{array}$ & 0.0 & 0.0 & 0.0 & 0.0 & 0.0 & 0.0 & 0.0 & 0.0 & 0.0 & 0.0 & 0.0 & 0.0 & 0.0 & 0.0 & 0.0 & 0.0 & 0.0 & 0.0 \\
\hline $\begin{array}{l}\text { Cervix uteri } \\
\text { cancer }\end{array}$ & 0.0 & 0.0 & 0.0 & 0.0 & 0.0 & 0.0 & 0.0 & 0.0 & 0.0 & 0.0 & 0.0 & 0.0 & 0.0 & 0.0 & 0.0 & 0.0 & 0.0 & 0.0 \\
\hline $\begin{array}{l}\text { Corpus uteri } \\
\text { cancer }\end{array}$ & 0.0 & 0.0 & 0.0 & 0.0 & 0.0 & 0.0 & 0.0 & 0.0 & 0.0 & 0.0 & 0.0 & 0.0 & 0.0 & 0.0 & 0.0 & 0.0 & 0.0 & 0.0 \\
\hline Ovary cancer & 0.0 & 0.0 & 0.0 & 0.0 & 0.0 & 0.0 & 0.0 & 0.0 & 0.0 & 0.0 & 0.0 & 0.0 & 0.0 & 0.0 & 0.0 & 0.0 & 0.0 & 0.0 \\
\hline $\begin{array}{l}\text { Prostate } \\
\text { cancer }\end{array}$ & 550.2 & 36.5 & 28.2 & 165.7 & 46.0 & 8.6 & 3.2 & 2.7 & 139.1 & 15.2 & 0.8 & 22.3 & 13.4 & 29.6 & 28.1 & 9.5 & 1.2 & 0.0 \\
\hline $\begin{array}{l}\text { Bladder } \\
\text { cancer }\end{array}$ & 277.2 & 10.6 & 7.3 & 60.3 & 8.1 & 0.8 & 4.1 & 14.4 & 65.1 & 12.1 & 1.2 & 17.8 & 7.7 & 27.8 & 13.5 & 24.8 & 1.5 & 0.0 \\
\hline $\begin{array}{l}\text { Lymphomas, } \\
\text { multiple } \\
\text { myeloma }\end{array}$ & 238.5 & 20.3 & 18.6 & 36.2 & 13.4 & 2.8 & 6.5 & 5.2 & 39.3 & 7.8 & 1.3 & 7.4 & 13.3 & 36.5 & 11.8 & 15.2 & 2.6 & 0.2 \\
\hline Leukaemia & 195.2 & 6.3 & 9.2 & 20.6 & 13.1 & 2.9 & 3.4 & 5.3 & 25.6 & 6.6 & I.I & 9.3 & 10.7 & 28.2 & 6.7 & 44.0 & 2.2 & 0.1 \\
\hline $\begin{array}{l}\text { Other } \\
\text { malignant } \\
\text { neoplasms }\end{array}$ & 635.8 & 24.2 & 33.5 & 84.8 & 54.2 & 8.6 & 10.3 & 6.2 & 128.4 & 32.5 & 4.7 & 56.3 & 24.2 & 77.8 & 35.8 & 47.7 & 6.4 & 0.3 \\
\hline
\end{tabular}

\begin{tabular}{|c|c|c|c|c|c|c|c|c|c|c|c|c|c|c|c|c|c|c|}
\hline \multicolumn{19}{|c|}{ Age-standardised incidence rate per 100,000} \\
\hline $\begin{array}{l}\text { Mouth and } \\
\text { oropharynx } \\
\text { cancers }\end{array}$ & 8.8 & 12.9 & 18.4 & 5.0 & 5.5 & 3.3 & 4.9 & 3.6 & 8.6 & 7.4 & 4.5 & 10.9 & 9.3 & 18.5 & 4.7 & 3.9 & 12.0 & 21.7 \\
\hline $\begin{array}{l}\text { Oesophagus } \\
\text { cancer }\end{array}$ & 9.4 & 5.7 & 16.8 & 5.1 & 5.9 & 3.1 & 3.8 & 1.7 & 6.0 & 3.4 & 9.4 & 6.3 & 2.2 & 8.2 & 6.6 & 19.2 & 6.3 & 2.0 \\
\hline $\begin{array}{l}\text { Stomach } \\
\text { cancer }\end{array}$ & 19.4 & 15.0 & 13.0 & 4.6 & 15.8 & 35.8 & 17.4 & 5.5 & 11.0 & 16.4 & 21.3 & 28.8 & 5.2 & 5.9 & 25.6 & 40.8 & 15.9 & 3.8 \\
\hline $\begin{array}{l}\text { Colon and } \\
\text { rectum } \\
\text { cancers }\end{array}$ & 17.3 & 10.4 & 14.6 & 34.4 & 11.3 & 9.2 & 6.3 & 6.5 & 33.0 & 20.4 & 7.1 & 25.1 & 12.9 & 4.9 & 32.4 & 12.3 & 7.8 & 5.8 \\
\hline Liver cancer & 13.7 & 20.3 & 24.7 & 3.7 & 5.0 & 11.4 & 6.4 & 5.0 & 6.4 & 5.7 & 3.6 & 4.8 & 16.0 & 3.2 & 16.2 & 30.8 & 12.1 & 9.9 \\
\hline $\begin{array}{l}\text { Pancreas } \\
\text { cancer }\end{array}$ & 3.9 & 1.6 & 3.5 & 6.5 & 4.3 & 4.8 & 1.8 & 2.3 & 6.8 & 5.7 & 5.3 & 6.8 & 2.2 & 1.5 & 7.3 & 2.5 & 1.0 & 0.2 \\
\hline $\begin{array}{l}\text { Trachea, } \\
\text { bronchus, } \\
\text { lung cancers }\end{array}$ & 30.9 & 8.3 & 12.0 & 44.8 & 20.6 & 6.9 & 18.9 & 16.6 & 43.3 & 47.1 & 20.6 & 52.2 & 27.5 & 20.3 & 28.9 & 30.8 & 26.4 & 14.7 \\
\hline $\begin{array}{l}\text { Melanoma of } \\
\text { the skin }\end{array}$ & 3.9 & 3.8 & 3.7 & 27.2 & 2.8 & 3.7 & 0.6 & 0.3 & 6.6 & 3.1 & 1.6 & 2.6 & 0.7 & 0.4 & 4.9 & 0.3 & 0.3 & 3.6 \\
\hline
\end{tabular}


Table 14: Estimated global and regional number of new cases and age-standardised incidence rate of cancer by site, males, 2000 (Continued)

\begin{tabular}{|c|c|c|c|c|c|c|c|c|c|c|c|c|c|c|c|c|c|c|}
\hline $\begin{array}{l}\text { Breast } \\
\text { cancer }\end{array}$ & 0.0 & 0.0 & 0.0 & 0.0 & 0.0 & 0.0 & 0.0 & 0.0 & 0.0 & 0.0 & 0.0 & 0.0 & 0.0 & 0.0 & 0.0 & 0.0 & 0.0 & 0.0 \\
\hline $\begin{array}{l}\text { Cervix uteri } \\
\text { cancer }\end{array}$ & 0.0 & 0.0 & 0.0 & 0.0 & 0.0 & 0.0 & 0.0 & 0.0 & 0.0 & 0.0 & 0.0 & 0.0 & 0.0 & 0.0 & 0.0 & 0.0 & 0.0 & 0.0 \\
\hline $\begin{array}{l}\text { Corpus uteri } \\
\text { cancer }\end{array}$ & 0.0 & 0.0 & 0.0 & 0.0 & 0.0 & 0.0 & 0.0 & 0.0 & 0.0 & 0.0 & 0.0 & 0.0 & 0.0 & 0.0 & 0.0 & 0.0 & 0.0 & 0.0 \\
\hline Ovary cancer & 0.0 & 0.0 & 0.0 & 0.0 & 0.0 & 0.0 & 0.0 & 0.0 & 0.0 & 0.0 & 0.0 & 0.0 & 0.0 & 0.0 & 0.0 & 0.0 & 0.0 & 0.0 \\
\hline $\begin{array}{l}\text { Prostate } \\
\text { cancer }\end{array}$ & 18.0 & 45.0 & 38.9 & 63.7 & 24.8 & 36.3 & 6.9 & 6.5 & 31.8 & 14.1 & 4.4 & 12.8 & 8.6 & 5.8 & 17.1 & 1.3 & 2.5 & 2.3 \\
\hline $\begin{array}{l}\text { Bladder } \\
\text { cancer }\end{array}$ & 9.0 & 12.0 & 8.8 & 24.4 & 4.3 & 3.3 & 8.5 & 30.5 & 16.1 & 11.6 & 6.4 & 10.7 & 4.6 & 4.8 & 8.7 & 3.5 & 3.1 & 1.4 \\
\hline $\begin{array}{l}\text { Lymphomas, } \\
\text { multiple } \\
\text { myeloma }\end{array}$ & 7.8 & 18.2 & 17.0 & 15.3 & 6.8 & 10.7 & 10.6 & 8.6 & 10.9 & 8.1 & 6.2 & 5.1 & 7.6 & 6.1 & 8.2 & 2.1 & 4.5 & 7.1 \\
\hline Leukaemia & 6.4 & 5.5 & 7.7 & 8.9 & 6.3 & 8.6 & 5.1 & 7.8 & 7.5 & 7.2 & 4.3 & 6.8 & 5.7 & 4.3 & 5.8 & 6.7 & 3.3 & 3.3 \\
\hline $\begin{array}{l}\text { Other } \\
\text { malignant } \\
\text { neoplasms }\end{array}$ & 20.8 & 24.1 & 37.1 & 39.9 & 27.2 & 31.0 & 19.1 & 11.3 & 39.1 & 32.8 & 22.4 & 37.5 & 13.5 & 13.7 & 27.0 & 6.6 & 12.4 & 11.2 \\
\hline
\end{tabular}

Table 15: Estimated global and regional number of new cases and age-standardised incidence rate of cancer by site, females, 2000

\begin{tabular}{|c|c|c|c|c|c|c|c|c|c|c|c|c|c|c|c|c|c|c|}
\hline \multirow[b]{3}{*}{ Site } & \multirow{3}{*}{ World } & \multicolumn{17}{|c|}{ WHO sub region } \\
\hline & & \multicolumn{2}{|c|}{ AFRO } & \multicolumn{3}{|c|}{ AMRO } & \multicolumn{2}{|c|}{ EMRO } & \multicolumn{4}{|c|}{ EURO } & \multicolumn{2}{|c|}{ SEARO } & \multicolumn{4}{|c|}{ WPRO } \\
\hline & & $\mathrm{D}$ & $E$ & A & B & $\mathrm{D}$ & B & $D$ & A & $\mathrm{BI}$ & B2 & C & B & $\mathrm{D}$ & $A$ & $\mathrm{BI}$ & B2 & B3 \\
\hline \multicolumn{19}{|c|}{ Number of new cases $(000 \mathrm{~s})$} \\
\hline $\begin{array}{l}\text { Mouth and } \\
\text { oropharynx } \\
\text { concers }\end{array}$ & 153.6 & 8.2 & 11.4 & 5.9 & 4.6 & 0.8 & 2.2 & I.I & 9.9 & 2.5 & 0.5 & 4.6 & 9.4 & 63.6 & 3.0 & 21.2 & 4.2 & 0.4 \\
\hline $\begin{array}{l}\text { Oesophagus } \\
\text { cancer }\end{array}$ & 166.2 & 4.2 & 7.2 & 3.3 & 4.0 & 0.3 & 1.6 & 0.5 & 8.4 & 1.0 & 1.3 & 3.8 & 2.5 & 34.9 & 2.1 & 89.0 & 2.0 & 0.0 \\
\hline $\begin{array}{l}\text { Stomach } \\
\text { cancer }\end{array}$ & 383.9 & 10.3 & 8.4 & 7.1 & 19.7 & 8.4 & 4.5 & 2.0 & 33.9 & 10.2 & 2.6 & 36.3 & 6.7 & 18.1 & 23.7 & 186.7 & 5.1 & 0.1 \\
\hline $\begin{array}{l}\text { Colon and } \\
\text { rectum } \\
\text { cancers }\end{array}$ & 517.3 & 8.5 & 8.2 & 73.8 & 26.2 & 3.3 & 4.0 & 2.8 & 131.0 & 19.0 & 1.8 & 54.1 & 22.8 & 21.1 & 41.7 & 95.5 & 3.3 & 0.1 \\
\hline Liver cancer & 204.4 & 11.9 & II.I & 4.7 & 9.7 & 3.4 & 1.3 & 1.2 & 14.0 & 4.0 & 0.5 & 5.9 & 11.7 & 12.0 & II.I & 99.6 & 2.2 & 0.2 \\
\hline $\begin{array}{l}\text { Pancreas } \\
\text { cancer }\end{array}$ & 106.9 & 1.6 & 2.6 & 14.3 & 9.1 & 1.3 & 0.8 & 0.7 & 28.1 & 4.8 & 0.7 & 10.2 & 3.6 & 5.3 & 9.8 & 13.6 & 0.5 & 0.0 \\
\hline $\begin{array}{l}\text { Trachea, } \\
\text { bronchus, } \\
\text { lung cancers }\end{array}$ & 365.7 & 3.1 & 5.0 & 74.6 & 16.4 & 1.0 & 3.0 & 2.6 & 55.3 & 10.8 & I.I & 17.7 & 8.3 & 25.9 & 18.9 & 117.7 & 4.0 & 0.2 \\
\hline $\begin{array}{l}\text { Melanoma of } \\
\text { the skin }\end{array}$ & 88.5 & 3.3 & 4.3 & 26.5 & 5.7 & 0.9 & 0.4 & 0.3 & 26.5 & 3.5 & 0.3 & 5.9 & 1.9 & 1.7 & 5.0 & 2.2 & 0.1 & 0.1 \\
\hline $\begin{array}{l}\text { Breast } \\
\text { cancer }\end{array}$ & 1031.9 & 28.3 & 39.8 & 226.5 & 59.3 & 7.2 & 10.2 & 13.6 & 221.8 & 32.6 & 4.5 & 68.5 & 53.9 & 109.0 & 43.7 & 102.1 & 10.2 & 0.5 \\
\hline $\begin{array}{l}\text { Cervix uteri } \\
\text { cancer }\end{array}$ & 487.5 & 43.3 & 67.9 & 16.1 & 40.6 & 9.2 & 6.7 & 5.6 & 18.8 & 12.6 & 1.9 & 22.5 & 32.8 & 146.7 & 6.6 & 45.0 & 10.2 & 0.8 \\
\hline $\begin{array}{l}\text { Corpus uteri } \\
\text { cancer }\end{array}$ & 326.3 & 4.6 & 6.0 & 46.0 & 48.8 & 8.6 & 1.6 & 1.3 & 68.3 & 17.9 & 3.8 & 43.6 & 19.9 & 11.7 & 17.3 & 17.4 & 0.8 & 0.1 \\
\hline $\begin{array}{l}\text { Ovary } \\
\text { cancer }\end{array}$ & 221.3 & 7.1 & 12.2 & 22.4 & 13.2 & 2.1 & 2.1 & 2.0 & 39.4 & 10.0 & 1.2 & 21.6 & 18.1 & 31.9 & 8.4 & 26.6 & 2.9 & 0.2 \\
\hline $\begin{array}{l}\text { Prostate } \\
\text { cancer }\end{array}$ & 0.0 & 0.0 & 0.0 & 0.0 & 0.0 & 0.0 & 0.0 & 0.0 & 0.0 & 0.0 & 0.0 & 0.0 & 0.0 & 0.0 & 0.0 & 0.0 & 0.0 & 0.0 \\
\hline $\begin{array}{l}\text { Bladder } \\
\text { cancer }\end{array}$ & 105.7 & 3.6 & 3.4 & 14.9 & 3.5 & 0.4 & 0.8 & 3.7 & 21.4 & 2.7 & 0.3 & 4.4 & 2.4 & 28.9 & 4.6 & 9.7 & 1.0 & 0.0 \\
\hline
\end{tabular}


Table 15: Estimated global and regional number of new cases and age-standardised incidence rate of cancer by site, females, 2000 (Continued)

\begin{tabular}{|c|c|c|c|c|c|c|c|c|c|c|c|c|c|c|c|c|c|c|}
\hline $\begin{array}{l}\text { Lymphomas, } \\
\text { multiple } \\
\text { myeloma }\end{array}$ & 242.6 & 10.2 & 8.0 & 32.2 & 12.4 & 2.3 & 5.0 & 4.0 & 40.9 & 6.9 & 1.0 & 7.3 & 9.6 & 74.9 & 9.7 & 15.2 & 2.7 & 0.1 \\
\hline Leukaemia & 169.5 & 5.9 & 7.0 & 14.7 & 12.4 & 2.9 & 2.6 & 3.8 & 23.0 & 4.9 & 0.9 & 9.0 & 10.8 & 24.9 & 5.2 & 39.4 & 1.9 & 0.1 \\
\hline $\begin{array}{l}\text { Other malig- } \\
\text { nant neo- } \\
\text { plasms }\end{array}$ & 553.9 & 44.2 & 22.7 & 63.6 & 56.7 & 11.7 & 8.9 & 8.9 & 107.3 & 25.3 & 4.4 & 46.5 & 27.3 & 31.7 & 32.7 & 56.1 & 5.3 & 0.3 \\
\hline \multicolumn{19}{|c|}{ Age-standardised incidence rate per 100,000} \\
\hline $\begin{array}{l}\text { Mouth and } \\
\text { oropharynx } \\
\text { cancers }\end{array}$ & 5.1 & 9.2 & 12.6 & 2.6 & 2.4 & 3.4 & 4.6 & 2.2 & 2.7 & 2.5 & 2.5 & 2.2 & 5.7 & 13.5 & 2.2 & 3.1 & 7.7 & 18.6 \\
\hline $\begin{array}{l}\text { Oesophagus } \\
\text { cancer }\end{array}$ & 5.6 & 5.2 & 9.2 & 1.3 & 2.2 & 1.5 & 4.0 & 1.1 & 1.8 & 1.0 & 6.5 & 1.5 & 1.7 & 8.0 & 1.3 & 13.4 & 3.9 & 1.8 \\
\hline $\begin{array}{l}\text { Stomach } \\
\text { cancer }\end{array}$ & 12.8 & 12.7 & 10.5 & 2.9 & 10.7 & 37.4 & 10.9 & 4.3 & 7.3 & 9.4 & 12.4 & 15.6 & 4.4 & 4.3 & 14.9 & 28.2 & 10.1 & 3.1 \\
\hline $\begin{array}{l}\text { Colon and } \\
\text { rectum } \\
\text { cancers }\end{array}$ & 17.3 & 9.9 & 10.2 & 29.8 & 14.1 & 14.4 & 9.4 & 5.9 & 29.2 & 17.2 & 8.6 & 23.0 & 14.7 & 4.7 & 26.7 & 14.2 & 6.3 & 4.2 \\
\hline Liver cancer & 6.8 & 13.0 & 12.0 & 1.9 & 5.2 & 14.6 & 3.1 & 2.5 & 3.1 & 3.6 & 2.3 & 2.5 & 7.5 & 2.3 & 6.6 & 14.8 & 4.2 & 7.6 \\
\hline $\begin{array}{l}\text { Pancreas } \\
\text { cancer }\end{array}$ & 3.6 & 1.8 & 3.5 & 5.6 & 5.0 & 5.8 & 1.9 & 1.6 & 6.0 & 4.3 & 3.6 & 4.3 & 2.4 & 1.3 & 5.8 & 2.1 & 0.9 & 0.4 \\
\hline $\begin{array}{l}\text { Trachea, } \\
\text { bronchus, } \\
\text { lung cancers }\end{array}$ & 11.9 & 3.5 & 5.8 & 30.5 & 8.8 & 4.4 & 6.7 & 5.6 & 13.4 & 9.9 & 5.5 & 7.6 & 5.4 & 5.3 & 11.6 & 16.0 & 7.4 & 8.4 \\
\hline $\begin{array}{l}\text { Melanoma of } \\
\text { the skin }\end{array}$ & 2.9 & 3.9 & 5.3 & 12.6 & 2.9 & 4.0 & 0.8 & 0.6 & 7.9 & 3.4 & 1.4 & 3.2 & 1.2 & 0.3 & 4.2 & 0.3 & 0.2 & 2.8 \\
\hline $\begin{array}{l}\text { Breast } \\
\text { cancer }\end{array}$ & 34.2 & 29.9 & 41.6 & 103.5 & 29.7 & 27.6 & 22.5 & 26.2 & 64.1 & 32.0 & 21.2 & 35.4 & 30.7 & 20.9 & 38.6 & 14.1 & 18.5 & 21.9 \\
\hline $\begin{array}{l}\text { Cervix uteri } \\
\text { cancer }\end{array}$ & 16.6 & 43.3 & 69.8 & 8.2 & 19.8 & 34.9 & 15.0 & 10.7 & 6.2 & 12.8 & 9.0 & 12.7 & 18.4 & 30.2 & 6.0 & 6.6 & 18.0 & 33.1 \\
\hline $\begin{array}{l}\text { Corpus uteri } \\
\text { cancer }\end{array}$ & 10.8 & 5.2 & 6.8 & 20.3 & 24.5 & 32.8 & 3.7 & 2.8 & 18.4 & 17.1 & 18.1 & 21.2 & 11.5 & 2.1 & 13.3 & 2.4 & 1.5 & 5.2 \\
\hline $\begin{array}{l}\text { Ovary } \\
\text { cancer }\end{array}$ & 7.3 & 6.8 & 12.1 & 9.8 & 6.6 & 7.9 & 4.3 & 3.7 & 10.8 & 9.9 & 5.6 & 11.2 & 10.2 & 6.2 & 6.8 & 3.6 & 5.0 & 6.3 \\
\hline $\begin{array}{l}\text { Prostate } \\
\text { cancer }\end{array}$ & 0.0 & 0.0 & 0.0 & 0.0 & 0.0 & 0.0 & 0.0 & 0.0 & 0.0 & 0.0 & 0.0 & 0.0 & 0.0 & 0.0 & 0.0 & 0.0 & 0.0 & 0.0 \\
\hline $\begin{array}{l}\text { Bladder } \\
\text { cancer }\end{array}$ & 3.6 & 4.1 & 3.9 & 6.0 & 1.9 & 1.8 & 2.2 & 7.9 & 4.6 & 2.4 & 1.5 & 1.9 & 1.6 & 5.7 & 2.7 & 1.5 & 1.8 & 0.6 \\
\hline $\begin{array}{l}\text { Lymphomas, } \\
\text { multiple } \\
\text { myeloma }\end{array}$ & 8.3 & 9.1 & 7.7 & 13.8 & 6.3 & 9.2 & 9.9 & 7.2 & 10.6 & 7.0 & 4.4 & 4.0 & 5.8 & 15.7 & 6.7 & 2.2 & 4.4 & 4.8 \\
\hline Leukaemia & 5.5 & 5.6 & 6.1 & 6.6 & 6.0 & 9.1 & 4.3 & 5.9 & 6.0 & 5.2 & 3.8 & 5.2 & 6.0 & 3.9 & 4.4 & 5.8 & 3.0 & 2.9 \\
\hline $\begin{array}{l}\text { Other } \\
\text { malignant } \\
\text { neoplasms }\end{array}$ & 18.8 & 45.9 & 24.0 & 30.2 & 28.6 & 44.0 & 20.0 & 17.2 & 29.6 & 24.9 & 19.6 & 25.8 & 16.1 & 6.1 & 23.3 & 10.7 & 9.4 & 129 \\
\hline
\end{tabular}

Table 16: Ranking of the global cancer incidence by site, 2000

\begin{tabular}{lcr}
\hline site & Number of death (000s) & Proportion of to \\
\hline Both sexes & & 12.7 \\
Trachea, bronchus, and lung & 1305.6 & 10.2 \\
Stomach & 1045.0 & 10.0 \\
Liver & 1031.9 & 9.5 \\
Colon and rectum & 973.1 & 6.1 \\
Breast & 626.4 & 5.3 \\
Oesophagus & 550.2 & 4.7 \\
Lymphomas and myeloma & 487.5 & 4.7 \\
Mouth and oropharynx & 481.1 & 4
\end{tabular}


Table 16: Ranking of the global cancer incidence by site, 2000 (Continued)

\begin{tabular}{|c|c|c|}
\hline Prostate & 452.7 & 4.4 \\
\hline Leukaemia & 420.7 & 4.1 \\
\hline Cervix uteri & 382.9 & 3.7 \\
\hline Pancreas & 364.7 & 3.5 \\
\hline Bladder & 326.3 & 3.2 \\
\hline Ovary & 226.2 & 2.2 \\
\hline Corpus uteri & 221.3 & 2.2 \\
\hline Melanoma of the skin & 206.7 & 2.0 \\
\hline \multicolumn{3}{|l|}{ Males } \\
\hline Trachea, bronchus, and lung & 939.9 & 18.2 \\
\hline Stomach & 589.3 & 11.4 \\
\hline Liver & 550.2 & 10.6 \\
\hline Colon and rectum & 527.8 & 10.2 \\
\hline Oesophagus & 422.0 & 8.2 \\
\hline Prostate & 286.5 & 5.5 \\
\hline Mouth and oropharynx & 277.2 & 5.4 \\
\hline Lymphomas and myeloma & 267.1 & 5.2 \\
\hline Leukaemia & 238.5 & 4.6 \\
\hline Bladder & 195.2 & 3.8 \\
\hline Pancreas & 119.3 & 2.3 \\
\hline Melanoma of the skin & 118.2 & 2.3 \\
\hline \multicolumn{3}{|l|}{ Females } \\
\hline Breast & 1031.9 & 20.1 \\
\hline Trachea, bronchus, and lung & 517.3 & 10.1 \\
\hline Stomach & 487.5 & 9.5 \\
\hline Colon and rectum & 383.9 & 7.5 \\
\hline Cervix uteri & 365.7 & 7.1 \\
\hline Liver & 326.3 & 6.4 \\
\hline Lymphomas and myeloma & 242.6 & 4.7 \\
\hline Oesophagus & 221.3 & 4.3 \\
\hline Ovary & 204.4 & 4.0 \\
\hline Leukaemia & 169.5 & 3.3 \\
\hline Pancreas & 166.2 & 3.2 \\
\hline Mouth and oropharynx & 153.6 & 3.0 \\
\hline Corpus uteri & 106.9 & 2.1 \\
\hline Bladder & 105.7 & 2.1 \\
\hline Melanoma of the skin & 88.5 & 1.7 \\
\hline
\end{tabular}

Table 17: Ranking of the selected regional cancer incidence by site, 2000

\begin{tabular}{|c|c|c|c|c|c|c|c|c|}
\hline \multicolumn{9}{|c|}{ WHO sub region } \\
\hline \multicolumn{3}{|c|}{ AfrE (high child and adult mortality) } & \multicolumn{3}{|c|}{ EurA (very low child and adult mortality) } & \multicolumn{3}{|c|}{ SearB (low child and adult mortality) } \\
\hline \multirow[t]{2}{*}{ site } & \multicolumn{2}{|c|}{ Number of Proportion } & \multirow[t]{2}{*}{ site } & \multicolumn{2}{|c|}{ Number of Proportion } & \multirow[t]{2}{*}{ site } & \multicolumn{2}{|c|}{ Number of Proportion } \\
\hline & $(000 s)$ & $(\%)$ & & $(000 s)$ & $(\%)$ & & $(000 s)$ & $(\%)$ \\
\hline \multicolumn{9}{|l|}{ Both sexes } \\
\hline Cervix uteri & 67.9 & 16.4 & $\begin{array}{c}\text { Trachea, } \\
\text { bronchus, and } \\
\text { lung }\end{array}$ & 264.7 & 15.4 & $\begin{array}{c}\text { Trachea, } \\
\text { bronchus, and } \\
\text { lung }\end{array}$ & 53.9 & 12.3 \\
\hline Liver & 39.8 & 9.6 & $\begin{array}{l}\text { Colon and } \\
\text { rectum }\end{array}$ & 227.3 & 13.2 & Liver & 51.4 & 11.7 \\
\hline Breast & 34.7 & 8.3 & Breast & 221.8 & 12.9 & Breast & 44.3 & 10.1 \\
\hline $\begin{array}{l}\text { Mouth and } \\
\text { oropharynx }\end{array}$ & 28.2 & 6.8 & Prostate & 139.1 & 8.1 & $\begin{array}{l}\text { Colon and } \\
\text { rectum }\end{array}$ & 40.3 & 9.2 \\
\hline
\end{tabular}


Table 17: Ranking of the selected regional cancer incidence by site, 2000 (Continued)

\begin{tabular}{|c|c|c|c|c|c|c|c|c|}
\hline Oesophagus & 27.9 & 6.7 & Stomach & 86.5 & 5.0 & $\begin{array}{l}\text { Mouth and } \\
\text { oropharynx }\end{array}$ & 32.8 & 7.5 \\
\hline $\begin{array}{l}\text { Lymphomas } \\
\text { and myeloma }\end{array}$ & 26.6 & 6.4 & $\begin{array}{l}\text { Lymphomas } \\
\text { and myeloma }\end{array}$ & 80.2 & 4.7 & $\begin{array}{l}\text { Lymphomas } \\
\text { and myeloma }\end{array}$ & 25.4 & 5.8 \\
\hline Prostate & 21.2 & 5.1 & Pancreas & 79.6 & 4.6 & Cervix uteri & 22.9 & 5.2 \\
\hline Stomach & 20.3 & 4.9 & Liver & 68.3 & 4.0 & Leukaemia & 21.5 & 4.9 \\
\hline $\begin{array}{l}\text { Colon and } \\
\text { rectum }\end{array}$ & 19.4 & 4.7 & Bladder & 55.5 & 3.2 & Stomach & 19.9 & 4.5 \\
\hline $\begin{array}{c}\text { Trachea, } \\
\text { bronchus, and } \\
\text { lung }\end{array}$ & 16.2 & 3.9 & Leukaemia & 49.1 & 2.8 & Ovary & 18.1 & 4.1 \\
\hline Leukaemia & 14.8 & 3.6 & Oesophagus & 48.6 & 2.8 & Prostate & 15.3 & 3.5 \\
\hline Ovary & 12.2 & 2.9 & Ovary & 40.1 & 2.3 & Pancreas & 13.4 & 3.1 \\
\hline Bladder & 10.7 & 2.6 & $\begin{array}{l}\text { Mouth and } \\
\text { oropharynx }\end{array}$ & 39.4 & 2.3 & Bladder & 10.1 & 2.3 \\
\hline $\begin{array}{l}\text { Melanoma of } \\
\text { the skin }\end{array}$ & 7.7 & 1.8 & Corpus uteri & 38.6 & 2.2 & Oesophagus & 7.2 & 1.7 \\
\hline Pancreas & 6.0 & 1.4 & $\begin{array}{l}\text { Melanoma of } \\
\text { the skin }\end{array}$ & 31.2 & 1.8 & Corpus uteri & 6.2 & 1.4 \\
\hline Corpus uteri & 5.4 & 1.3 & Cervix uteri & 18.8 & I.I & $\begin{array}{l}\text { Melanoma of } \\
\text { the skin }\end{array}$ & 3.1 & 0.7 \\
\hline \multicolumn{9}{|l|}{ Males } \\
\hline Liver & 28.2 & 14.8 & $\begin{array}{c}\text { Trachea, } \\
\text { bronchus, and } \\
\text { lung }\end{array}$ & 172.0 & 19.6 & $\begin{array}{c}\text { Trachea, } \\
\text { bronchus, and } \\
\text { lung }\end{array}$ & 43.1 & 22.0 \\
\hline Prostate & 23.6 & 12.4 & $\begin{array}{l}\text { Colon and } \\
\text { rectum }\end{array}$ & 139.1 & 15.9 & Liver & 28.6 & 14.6 \\
\hline $\begin{array}{l}\text { Mouth and } \\
\text { oropharynx }\end{array}$ & 18.6 & 9.8 & Prostate & 133.8 & 15.3 & $\begin{array}{l}\text { Colon and } \\
\text { rectum }\end{array}$ & 21.4 & 11.0 \\
\hline $\begin{array}{l}\text { Lymphomas } \\
\text { and myeloma }\end{array}$ & 16.5 & 8.7 & Stomach & 65.1 & 7.4 & $\begin{array}{l}\text { Mouth and } \\
\text { oropharynx }\end{array}$ & 16.0 & 8.2 \\
\hline Oesophagus & 14.1 & 7.4 & $\begin{array}{l}\text { Lymphomas } \\
\text { and myeloma }\end{array}$ & 45.6 & 5.2 & $\begin{array}{l}\text { Lymphomas } \\
\text { and myeloma }\end{array}$ & 13.4 & 6.9 \\
\hline Stomach & 12.0 & 6.3 & Bladder & 39.3 & 4.5 & Prostate & 13.3 & 6.8 \\
\hline $\begin{array}{c}\text { Trachea, } \\
\text { bronchus, and } \\
\text { lung }\end{array}$ & 10.9 & 5.8 & Pancreas & 28.7 & 3.3 & Leukaemia & 10.7 & 5.5 \\
\hline $\begin{array}{l}\text { Colon and } \\
\text { rectum }\end{array}$ & 9.8 & 5.2 & Liver & 27.4 & 3.1 & Stomach & 8.6 & 4.4 \\
\hline Leukaemia & 9.2 & 4.9 & Oesophagus & 26.1 & 3.0 & Bladder & 7.7 & 3.9 \\
\hline Bladder & 7.3 & 3.8 & Leukaemia & 25.6 & 2.9 & Pancreas & 3.8 & 1.9 \\
\hline Pancreas & 3.4 & 1.8 & $\begin{array}{l}\text { Mouth and } \\
\text { oropharynx }\end{array}$ & 22.8 & 2.6 & Oesophagus & 3.7 & 1.9 \\
\hline $\begin{array}{l}\text { Melanoma of } \\
\text { the skin }\end{array}$ & 2.8 & 1.5 & $\begin{array}{l}\text { Melanoma of } \\
\text { the skin }\end{array}$ & 22.6 & 2.6 & $\begin{array}{l}\text { Melanoma of } \\
\text { the skin }\end{array}$ & 1.2 & 0.6 \\
\hline \multicolumn{9}{|l|}{ Females } \\
\hline Cervix uteri & 67.9 & 30.2 & Breast & 221.8 & 26.2 & Breast & 53.9 & 22.3 \\
\hline Breast & 39.8 & 17.7 & $\begin{array}{l}\text { Colon and } \\
\text { rectum }\end{array}$ & 131.0 & 15.4 & Cervix uteri & 32.8 & 13.6 \\
\hline Liver & 12.2 & 5.4 & $\begin{array}{c}\text { Trachea, } \\
\text { bronchus, and } \\
\text { lung }\end{array}$ & 68.3 & 8.1 & $\begin{array}{l}\text { Colon and } \\
\text { rectum }\end{array}$ & 22.8 & 9.4 \\
\hline Stomach & 11.4 & 5.1 & Stomach & 55.3 & 6.5 & Liver & 19.9 & 8.2 \\
\hline $\begin{array}{l}\text { Mouth and } \\
\text { oropharynx }\end{array}$ & 11.1 & 4.9 & $\begin{array}{l}\text { Lymphomas } \\
\text { and myeloma }\end{array}$ & 40.9 & 4.8 & Ovary & 18.1 & 7.5 \\
\hline Ovary & 8.4 & 3.7 & Pancreas & 39.4 & 4.6 & $\begin{array}{c}\text { Trachea, } \\
\text { bronchus, and } \\
\text { lung }\end{array}$ & 11.7 & 4.8 \\
\hline
\end{tabular}


Table 17: Ranking of the selected regional cancer incidence by site, 2000 (Continued)

\begin{tabular}{|c|c|c|c|c|c|c|c|c|}
\hline Oesophagus & 8.2 & 3.7 & Ovary & 33.9 & 4.0 & Leukaemia & 10.8 & 4.5 \\
\hline $\begin{array}{l}\text { Colon and } \\
\text { rectum }\end{array}$ & 8.0 & 3.6 & Leukaemia & 28.1 & 3.3 & $\begin{array}{l}\text { Lymphomas } \\
\text { and myeloma }\end{array}$ & 9.6 & 4.0 \\
\hline $\begin{array}{l}\text { Lymphomas } \\
\text { and myeloma }\end{array}$ & 7.2 & 3.2 & Corpus uteri & 26.5 & 3.1 & $\begin{array}{l}\text { Mouth and } \\
\text { oropharynx }\end{array}$ & 9.4 & 3.9 \\
\hline Leukaemia & 7.0 & 3.1 & Liver & 23.0 & 2.7 & Stomach & 8.3 & 3.4 \\
\hline $\begin{array}{c}\text { Trachea, } \\
\text { bronchus, and } \\
\text { lung }\end{array}$ & 6.0 & 2.7 & Bladder & 21.4 & 2.5 & Corpus uteri & 6.7 & 2.8 \\
\hline $\begin{array}{l}\text { Melanoma of } \\
\text { the skin }\end{array}$ & 5.0 & 2.2 & Cervix uteri & 18.8 & 2.2 & Pancreas & 3.6 & 1.5 \\
\hline Pancreas & 4.3 & 1.9 & Oesophagus & 14.0 & 1.7 & Oesophagus & 2.5 & 1.0 \\
\hline Bladder & 3.4 & 1.5 & $\begin{array}{l}\text { Melanoma of } \\
\text { the skin }\end{array}$ & 9.9 & 1.2 & Bladder & 2.4 & 1.0 \\
\hline Corpus uteri & 2.6 & 1.2 & $\begin{array}{l}\text { Mouth and } \\
\text { oropharynx }\end{array}$ & 8.4 & 1.0 & $\begin{array}{l}\text { Melanoma of } \\
\text { the skin }\end{array}$ & 1.9 & 0.8 \\
\hline
\end{tabular}

ture, distribution of risk factors, and opportunity of detection and treatment $[2,22]$. However, the frequent cancers such as lung, liver and cervical cancers are potentially preventable [23]. For example, Parkin has estimated that there would have been $23 \%$ fewer cases of cancers in the developing world in 1990, if infections such as hepatitis $\mathrm{B}$ and $\mathrm{C}$ virus and human papilloma virus had been prevented [24]. Another estimate suggests that 230,000 deaths (more than $4 \%$ of all cancer deaths) from liver cancer could have been avoided with only immunisations against hepatitis B [13]. Smoking was estimated to be responsible for another $20 \%$ of all cancer deaths, all of which are preventable [13].

Given a high incidence of cancers which are potentially preventable in both developed and developing countries, the role of primary prevention, early detection as well as treatment should be evaluated more carefully [2]. Cost-effectiveness analysis plays a role for this purpose, which should be generalisable and comparable across various interventions including both currently delivered and potentially feasible ones [20]. Estimating the magnitude of cancer mortality and incidence is a key input for setting research and intervention priorities. Combined with costs of each intervention, mortality and incidence estimates provide a basis for effectiveness calculation in cost-effectiveness analysis of cancer control programmes.

\section{Conclusions}

Based on the algorithm to estimate region-specific overall cancer mortality, and site-specific survival, death distributions, and incidence as a part of GBD 2000 study, it is estimated that cancers accounted for over 7 million deaths (13\% of total mortality) and more than 10 million new cases occurred world wide in 2000. Cancer mortality and incidence in developing countries already accounted for over $60 \%$ and about half of the global total, respectively.
Although there was a significant variation in the distribution of cancer mortality and incidence by region, many of the common cancers are potentially preventable. Magnitude of cancer burden estimation by taking into account both mortality and morbidity is an essential information to set research priorities and policy formulation and can be used for setting cancer control priorities when combined with data on costs of interventions.

\section{Competing interests}

None declared.

\section{Author's Contributions}

KS conceived of the study, analysed the data, and drafted the manuscript. CDM participated in the design of the study, and performed statistical analyses. CBP carried out the data compilation and drafted the manuscript. ADL participated in the design of the study and in the mortality analysis. CJLM conceived of the study and participated in its design and coordination. All authors read and approved the final manuscript.

\section{Acknowledgements}

Many people are contributing to the analysis of cancer incidence and mortality for the GBD 2000 both inside and outside WHO. We wish to particularly acknowledge the contributions of staff within the Global Program on Evidence for Health Policy who have contributed to the estimation of total cancer deaths for the year 2000: Majid Ezzati, Brodie Ferguson, Mie Inoue, Rafael Lozano, Doris Ma Fat, Chalapati Rao, Joshua Salomon, and Niels Tomijima. We also thank staff of the International Agency for Research on Cancer (IARC) for provision of data, advice on survival analyses carried out by IARC and methods used to estimate cancer incidence and mortality for GLOBOCAN 2000, particularly Max Parkin, Jacques Ferlay, Paola Pisani and Fred Bray.

\section{References}

I. Parkin DM, Pisani $P$ and Ferlay J Estimates of the worldwide incidence of 25 major cancers in 1990. Int J Cancer 1999, 80(6):827841 
2. Sankaranarayanan $R$, Swaminathan $R$ and Black $R$ J Global variations in cancer survival. Study Group on Cancer Survival in Developing Countries. Cancer 1996, 78:246I-2464

3. Graupera Boschmonar MC, Jimenez Chaviano PJ and Martin Garcia AA Trends in survival rates of cancer in Cuba. Eur J Epidemiol 1999, 15:521-528

4. Chia KS, Du WB and Sankaranarayanan R Population-based cancer survival in Singapore, 1968 to 1992: an overview. Int J Cancer 2001, 93:142-147

5. Murray CJL and Lopez AD The Global Burden of Disease. Cambridge, MA, Harvard University Press 1996,

6. Murray CIL, Lopez AD and Mathers CD The Global Burden of Disease $\mathbf{2 0 0 0}$ project: aims, methods and data sources. Geneva, World Health Organization 200I,

7. Mathers CD, Shibuya $K$ and Boschi-Pinto $C$ Global and regional estimates of cancer mortality and incidence by site: I. Application of regional cancer survival model to estimate cancer mortality distribution by site. BMC Cancer 2002, 36

8. Salomon JA and Murray CJL The epidemiological transition revisited: compositional models for causes of death by age and sex. Popul Dev Rev 2002, 28:205-228

9. Lopez $A D$, Ahmad $O$ and Guillot $M$ Life tables for 191 countries for 2000: data, methods, results. GPE Discussion Paper No. 40. Geneva, World Health Organization 200I,

10. Lopez $A D$, Ahmad $O$ and Guillot $M$ World mortality in 2000: life tables for I 9 I countries. Geneva, World Health Organization 2002

II. Bayo S, Parkin DM and Koumare AK Cancer in Mali, 1987-1988. Int J Cancer 1990, 45:679-684

12. Ferlay J, Bray F and Pisani P Globocan 2000: Cancer Incidence, Mortality and Prevalence Worldwide, Version I.0. IARC CancerBase No. 5. Lyon, IARCPress 200I,

13. Pisani P, Parkin DM and Bray F Estimates of the worldwide mortality from 25 cancers in 1990. Int J Cancer 1999, 83(1):18-29

14. Murray $C J L$ and Lopez $A D$ Mortality by cause for eight regions of the world: global burden of disease study. Lancet 1997, 349:1269-1276

15. Parkin DM The global burden of cancer. Semin Cancer Biol 1998 8:219-235

16. Gupta P, Sankaranarayanan R and Ferlay J Cancer death in India: is the model-based approach valid? Bull World Health Organ 1994, 72:943-944

17. Mosconi $\mathrm{P}, \mathrm{Apolone} \mathrm{G}$ and Barni S Quality of life in breast and coIon cancer long-term survivors: an assessment with the EORTC QLQ-C30 and SF-36 questionnaires. Tumori 2002, 88:110-116

18. Pandey $M$, Singh $S$ and Behere $P$ Quality of life in patients with early and advanced carcinoma of the breast. Eur J Surg Oncol 200I, 26:20-24

19. Bergmark K, Avall-Lundqvist E and Dickman PW Patient-rating of distressful symptoms after treatment for early cervical cancer. Acta Obstet Gynecol Scand 2002, 8 I:443-450

20. Murray CJ, Evans DB and Acharya A Development of WHO guidelines on generalized cost-effectiveness analysis. Health Econ 2000, 9:235-251

21. Üstün TB, Chatterji $S$ and Villanueva M WHO Multi-country Household Survey Study on Health and Responsiveness, 2000-200I. GPE discussion paper No. 37. Geneva, World Health Organization 200I,

22. Sankaranarayanan $R$, Swminathan $R$ and Black RJ Global variations in cancer survival. Cancer 1996, 78:246I-4

23. Doll $D$ and Peto $R$ The causes of cancer. Quantitative estimates of avoidable risks of cancer in the United States today. Oxford, Oxford University Press 1981,

24. Parkin DM The global health burden of infection associated cancers. Cancer Surv 1999, 33:5-33

\section{Pre-publication history}

The pre-publication history for this paper can be accessed here:

http://www.biomedcentral.com/1471-2407/2/37/prepub
Publish with Biomed Central and every scientist can read your work free of charge

"BioMed Central will be the most significant development for disseminating the results of biomedical research in our lifetime. "

Sir Paul Nurse, Cancer Research UK

Your research papers will be:

- available free of charge to the entire biomedical community

- peer reviewed and published immediately upon acceptance

- cited in PubMed and archived on PubMed Central

- yours - you keep the copyright
BioMedcentral 